\title{
Isotopic tracers of paleohydrologic change in large lakes of the Bolivian Altiplano
}

\author{
Christa J. Placzek $^{\mathrm{a}, *}$, Jay Quade ${ }^{\mathrm{b}}$, P. Jonathan Patchett ${ }^{\mathrm{b}}$ \\ ${ }^{a}$ Los Alamos National Laboratory, MS-J514, Los Alamos, NM 87545, USA \\ b Department of Geosciences, University of Arizona, Tucson, AZ 85721, USA
}

\section{A R T I C L E I N F O}

\section{Article history:}

Received 10 January 2010

Available online 19 September 2010

\section{Keywords:}

Altiplano

Lake

Climate

North Atlantic

ENSO

Bolivia

Paleolakes

Strontium

\begin{abstract}
A B S T R A C T
We have developed an ${ }^{87} \mathrm{Sr} /{ }^{86} \mathrm{Sr},{ }^{234} \mathrm{U} /{ }^{238} \mathrm{U}$, and $\delta^{18} \mathrm{O}$ data set from carbonates associated with late Quaternary paleolake cycles on the southern Bolivian Altiplano as a tool for tracking and understanding the causes of lake-level fluctuations. Distinctive groupings of ${ }^{87} \mathrm{Sr} /{ }^{86} \mathrm{Sr}$ ratios are observed. Ratios are highest for the Ouki lake cycle (120-95 ka) at 0.70932, lowest for Coipasa lake cycle (12.8-11.4 ka) at 0.70853, and intermediate at 0.70881 to 0.70884 for the Salinas (95-80 ka), Inca Huasi ( $45 \mathrm{ka})$, Sajsi (24-20.5 ka), and Tauca (18.1-14.1 ka) lake cycles. These $\mathrm{Sr}$ ratios reflect variable contributions from the eastern and western Cordilleras. The Laca hydrologic divide exerts a primary influence on modern and paleolake ${ }^{87} \mathrm{Sr} /{ }^{86} \mathrm{Sr}$ ratios; waters show higher ${ }^{87} \mathrm{Sr} /{ }^{86} \mathrm{Sr}$ ratios north of this divide. Most lake cycles were sustained by slightly more rainfall north of this divide but with minimal input from Lake Titicaca. The Coipasa lake cycle appears to have been sustained mainly by rainfall south of this divide. In contrast, the Ouki lake cycle was an expansive lake, deepest in the northern (Poópo) basin, and spilling southward. These results indicate that regional variability in central Andean wet events can be reconstructed using geochemical patterns from this lake system.
\end{abstract}

Published by Elsevier Inc. on behalf of University of Washington.

\section{Introduction}

Numerous paleolakes once occupied the Poópo, Coipasa, and Uyuni basins on the southern Bolivian Altiplano and provide dramatic evidence of major changes in low-latitude moisture. These were among the largest late Quaternary paleolakes in the Americas (30,000$60,000 \mathrm{~km}^{2}$ ), and span almost $3^{\circ}$ of latitude. The deposits associated with these lakes have been the focus of multiple investigations, including studies of cores (e.g. Sylvestre et al., 1999; Fornari et al., 2001; Baker et al., 2001a; Fritz et al., 2004) and shoreline deposits (e.g. Rondeau, 1990; Sylvestre et al., 1999; Placzek et al., 2006a) from a variety of perspectives, biological, geochemical, and geochronological. Interpretation of these archives has and will continue to make a significant contribution to our understanding of tropical climate change (e.g. Seltzer et al., 2003).

Strontium ( $\mathrm{Sr}$ ), uranium (U), and especially oxygen $(\mathrm{O})$ isotopes are widely used geochemical tools in lake studies for reconstructing paleohydrologic and hence paleoclimate changes. The uses and limitations of oxygen isotopes are well known, since important variables such as temperature, rainfall, and evaporation all influence, often in complex ways, the oxygen isotopic composition of lakes. Strontium and uranium isotopes are less widely employed in paleolake studies and yet can be extremely useful as stratigraphic tools (e.g. Hart et al., 2004; Ku et al., 1998), for constraining lake levels (Benson and

\footnotetext{
* Corresponding author. Fax: +1 5056654955.

E-mail address: cplaczek@lanl.gov (C.J. Placzek).
}

Peterman, 1995), and for reconstructing the history of lake overflow between sub-basins in large complex systems like the one under study here (Hart et al., 2004; Benson and Peterman, 1995).

For this study, our main focus is on the $\mathrm{Sr}$ system because individual lake cycles often have unique $\mathrm{Sr}$ isotopic ratios. As such, we are able to utilize these values as stratigraphic markers. Furthermore, we measure and then model the modern fluxes of $\mathrm{Sr}$ isotopes to contextualize results from ancient carbonates associated with the various paleolakes. Our U and O results add useful constraints on the Sr system. Both the Sr (Coudrain et al., 2002; Grove et al., 2003) and O (Cross et al., 2001 ) isotopic composition of this lake system have been studied to some degree already. We synthesize these sometimes contradictory results and add to them a large body of new results on both the modern and ancient lake systems. Among other things, this perspective allows us to speculate on possible changes in moisture sources to the lakes.

It is now apparent that two major lake expansions on the southern Bolivian Altiplano during the late Quaternary are part of a regional wet interval (18-8 ka) known as the Central Andean Pluvial Event (CAPE) (Latorre et al., 2006; Quade et al., 2008). The region impacted by CAPE appears to extend from 10 to $26^{\circ} \mathrm{S}$ (Latorre et al., 2006; Smith et al., 2005). Changes in North Atlantic sea-surface temperature (SST) gradients are implied as the principal driver of the CAPE (Zech et al., 2007; Blard et al., 2009). However, significant modern climate variability is observed across the broad region impacted by CAPE. For example, there are two modes of summer rainfall variability on the southern Bolivian, Altiplano, one sourced to the north-northeast and one derived from the southeast (Vuille and Keimig, 2004). Changes in the relative importance of these two modes of modern precipitation variability are 
proposed as the driver of regional asynchrony within the CAPE (Quade et al., 2008). One outcome of our present and ongoing studies is to better differentiate the relative contribution of these two rainfall modes under ancient conditions and ultimately to establish the connections of these rainfall modes to tropical and extratropical climatic changes.

Study area

The Altiplano is a high-elevation $(\sim 3800 \mathrm{~m})$, internally drained plateau. Four large lake basins (from North to South, respectively, Titicaca, Poópo, Coipasa, and Uyuni) occupy the region between the Western Cordillera (WC) and the Eastern Cordillera (EC), and are part of a watershed spanning $14^{\circ}$ to $22^{\circ} \mathrm{S}$ (Fig. 1 ). In this paper we consider the "southern Altiplano" to be the area encompassing all watersheds south of Lake Titicaca. The "northern Altiplano" is covered by Lake Titicaca (3806 m; $8560 \mathrm{~km}^{2}$ ), a deep (>285 m), fresh-water lake with minor $(<10 \%)$ outflow to the south along the Río Desaguadero into the

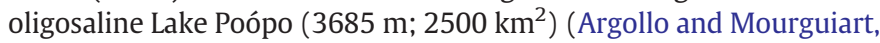

2000). By the time the Río Desaguadero reaches Lake Poópo it has more than quadrupled in size, and its water chemistry is notably different from that of Lake Titicaca (Grove et al., 2003). Lake Poópo is separated by the Laca hydrologic divide $(3700 \mathrm{~m})$ from the Salars (salt pans) of Coipasa $\left(3656 \mathrm{~m}, 2530 \mathrm{~km}^{2}\right)$ and Uyuni $(3653 \mathrm{~m}$, $12,000 \mathrm{~km}^{2}$ ) (Fig. 1). During exceptionally wet years (e.g. 1986) water flows over this hydrologic divide and into the Coipasa basin (Zolá and Bengtsson, 2007). The Río Laca Jahuira also originates here and flows west into the Coipasa basin (Fig. 1). The Río Lauca is the primary source of water for a small ephemeral lake in the Coipasa basin, and has a minimum estimated discharge of $0.2 \times 10^{9} \mathrm{~m}^{3} \mathrm{yr}^{-1}$ (Grove et al., 2003). The broad divide between the two salars in the Coipasa and Uyuni basins is only $1 \mathrm{~m}$ high and during flood events these salars form a single connected water body. For this reason, we consider the Coipasa and Uyuni basins to be a single basin during lake expansion, the Coipasa/Uyuni basin. The Río Grande is the only major watercourse entering the Uyuni basin and has an estimated annual discharge of $0.4 \times 10^{9} \mathrm{~m}^{3} \mathrm{yr}^{-1}$ (Montes de Octa, 1997).

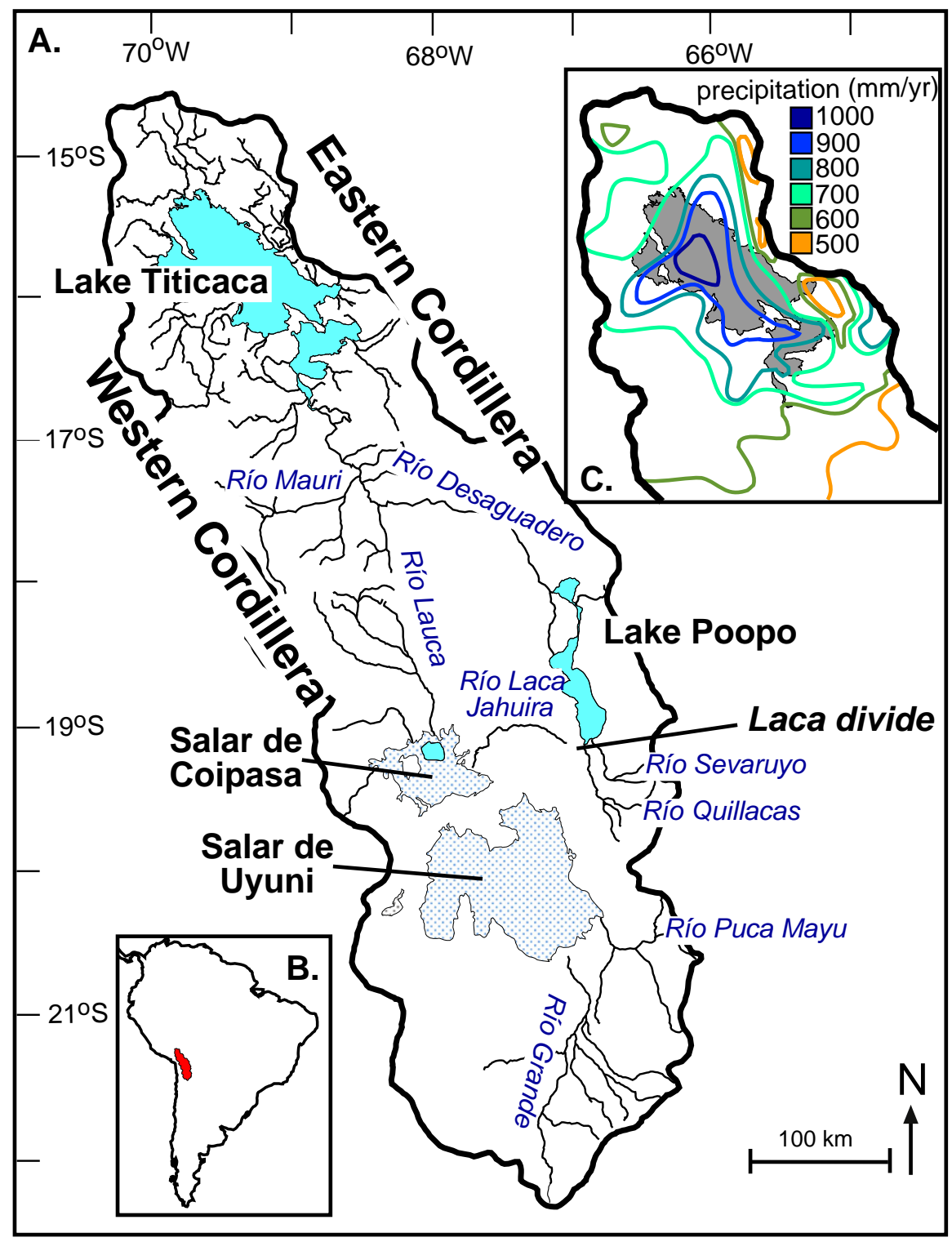

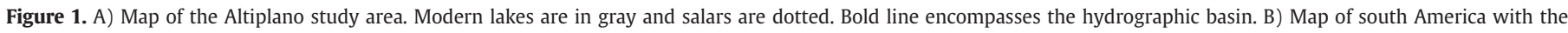

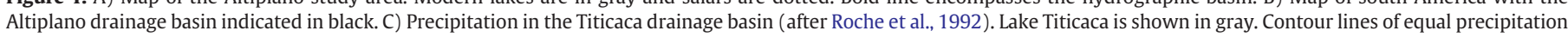
are shown in shades of black ( $1000 \mathrm{~mm} / \mathrm{yr}$ ) to light gray (500 mm/yr). 


\section{Modern climate}

The Bolivian Altiplano is located at the western end of the Amazon rainfall belt, and greater than $80 \%$ of rainfall occurs in the austral summer (e.g. Vuille, 1999) in what is described by many as the South American Summer Monsoon (SASM) (e.g. Zhou and Lau, 1998). A pronounced north-south precipitation gradient spans the TiticacaPoópo-Coipasa-Uyuni catchment, with Lake Titicaca receiving $\sim 800 \mathrm{~mm}$ of precipitation per year $(\mathrm{mm} / \mathrm{yr})$ and the dry southern basins receiving less than $200 \mathrm{~mm} / \mathrm{yr}$ (Fig. 2A). Two distinct modes of variability in modern summer rainfall occur over the central Andes, and these modes overlap each other on the southern Bolivian Altiplano (Vuille and Keimig, 2004). Rain falling on the northern Altiplano originates from the east and falls predominantly in the summer months when the Intertropical Convergence Zone is displaced southward and convection is most intense in the Amazon Basin (Lenters and Cook, 1997). This rainfall regime is modulated by several factors, including North Atlantic sea-surface temperature (Enfield and Mayer, 1997; Vuille et al., 2000), and El Niño/Southern Oscillation (ENSO), with La Niña years tending to correlate with stronger easterly winds and more rainfall (Aceituno, 1988; Vuille et al., 1998, Vuille, 1999; Garreaud and Aceituno, 2001; Vuille and Keimig, 2004). In contrast, summer rainfall on the southern Altiplano has interannual variability that is most closely tied to precipitation anomalies and humidity levels over the Chaco region of Argentina (Vuille and Keimig, 2004). Interplay between these two modes of modern climate variability may explain the spatial and temporal variability in a regional late Pleistocene wet event, the Central Andean Pluvial Event (CAPE) (Quade et al., 2008).

\section{Lake nomenclature and chronology}

Shoreline sediments and $>200$ radiometric ages place constraints on two high elevation, one mid-elevation, and three shallow lake

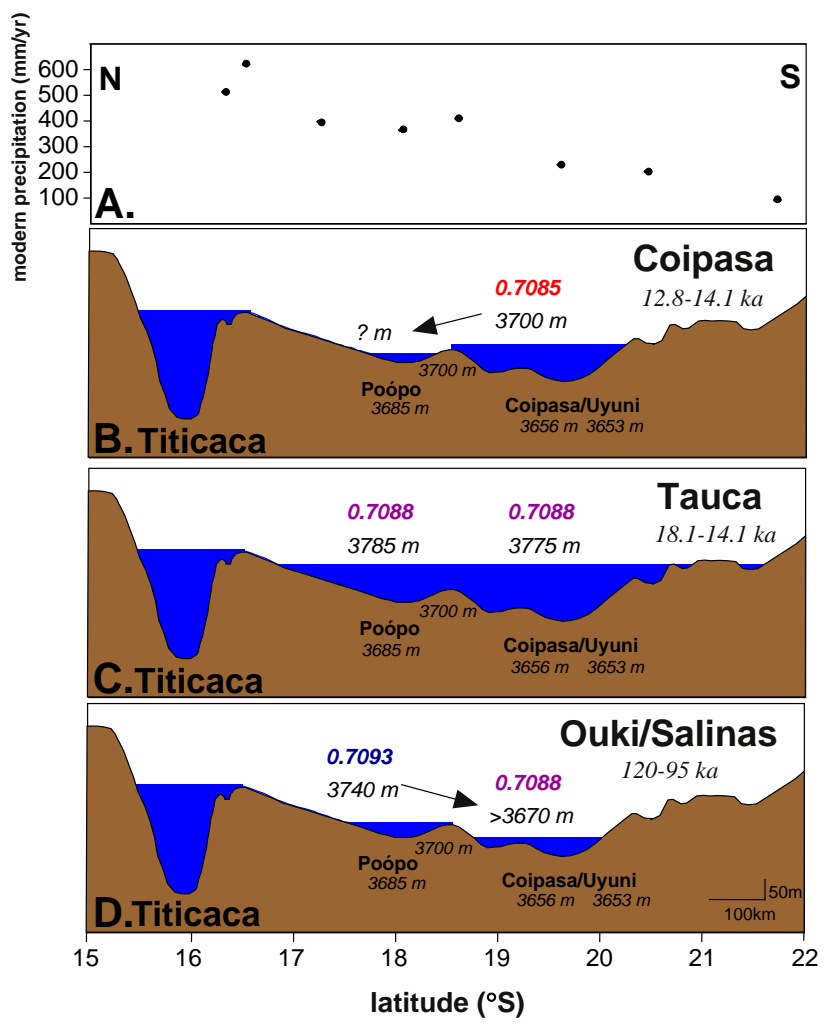

Figure 2. A) The modern precipitation gradient in the Titicaca, Poópo, and Coipasa/ Uyuni basins. Schematic cross section of the Titicaca/Poópo/Coipasa/Uyuni hydrographic basins and proposed flow between basins during the Coipasa (B), Tauca (C), and Ouki (D) lake cycles. Ratios of ${ }^{87} \mathrm{Sr} /{ }^{86} \mathrm{Sr}$ and lake elevations for each lake cycle are shown. cycles over the last $130 \mathrm{ka}$ in the Poópo, Coipasa, and Uyuni basins (Servant and Fontes, 1978; Rondeau, 1990; Bills et al., 1994; Sylvestre et al., 1999; Placzek et al., 2006a,b). These pluvial episodes have been of interest for over a century (e.g. Minchin, 1882), resulting in several, sometimes contradictory, names and chronologies for various lake cycles. In this paper we continue to use the nomenclature for these lake phases employed in our previous research (Fig. 3A; Placzek et al., 2006a,b). The Ouki lake cycle (120-95 ka) is dated at numerous localities in the Poópo basin and reached its maximum elevation (3740 $\mathrm{m}$ ) between 110 and $100 \mathrm{ka}$. A much shallower lake, the Salinas lake cycle $(<3670 \mathrm{~m})$, was present in the Uyuni Basin between 95 and $80 \mathrm{ka}$. No single outcrop contains evidence of a continuous lake over the entire Ouki and Salinas lake intervals (120-95 ka), but Placzek et al. (2006a) differentiate between lacustrine deposits with ages between $95-80 \mathrm{ka}$ and $120-95$ ka because they have different elevations in different basins. Examination of individual outcrops suggest that individual sections of the Ouki lake cycle may indeed record different phases of this lake; eventual correlation of shoreline and sediment core stratigraphies could lead to subdivision of the Ouki lake cycle into different sub-phases. Shallow lakes $(<3670 \mathrm{~m})$ were also present in the Uyuni basin around 46 ka (Inca Huasi lake cycle), and between 24 and $20.5 \mathrm{ka}$ (the Sajsi lake cycle). The Tauca lake cycle (18.1-14.1 ka) was the deepest lake on the Bolivian Altiplano in the last $120 \mathrm{ka}$ and reached its maximum $(\sim 3785 \mathrm{~m})$ between 16.4 and $14.1 \mathrm{ka}$. The Coipasa lake cycle occurred between 12.8 and $11.4 \mathrm{ka}$ (Placzek et al., 2006a).

\section{Methods}

Over 30 exposures of lacustrine sediments were described and sampled as part of a comprehensive effort to obtain and replicate records of lake change from multiple localities in all three major basins (Placzek et al., 2006a); sample sites from this study directly correspond to sites and samples described in these previous efforts. Particular effort was directed towards the various visible paleoshorelines, as this approach to reconstructing lake-level history allows for direct determination of lake level, replication of stratigraphy, and dating by two geochronologic $\left({ }^{14} \mathrm{C}, \mathrm{U}-\mathrm{Th}\right)$ methods. A firm U-Th chronology for the Tauca lake cycle (18.1-14.1 ka) indicates minimal hard-water effects on ${ }^{14} \mathrm{C}$ dates. For older samples (>25 ka), U-Th ages are significantly ( $>50 \mathrm{ka}$ ) older than associated ${ }^{14} \mathrm{C}$ dates, as a result of contamination by as much as $3 \%$ modern carbon. The cause for this is likely related to the surface exchange of atmospheric $\mathrm{CO}_{2}$ with carbonate radicals, a process observed when carbonates gradually take up $\mathrm{CO}_{2}$ after grinding (Samos, 1949). This contamination of the ${ }^{14} \mathrm{C}$ system is, therefore, not indicative of significant alteration of other isotopic systems; carbonate powders selected for this study are from tufas or shells that were free from secondary cement and low in noncarbonate detritus. Tufas from the southern Altiplano are ubiquitous features in the paleolake basins and have readily identified biologic textures (Placzek et al., 2006b) indicating formation within the photic zone. Sample locations in proximity to springs and/or the rare tufa with a form indicative of formation under the influence of groundwater input (e.g. chimney structures) were avoided in this sampling effort.

Waters and carbonate powders were sampled for $\mathrm{Sr}$ and $\mathrm{O}$ analyses. We collected water samples during June 2001 and again in NovemberDecember 2001; $50 \mathrm{ml}$ of water was passed through disposable $0.2 \mu \mathrm{m}$ glass syringe filters and stored in sealed acid-washed polyethylene bottles. Samples of water and salt were also collected from the Salars of Coipasa and Uyuni in June 2001. Carbonate samples were soaked in 2\% $\mathrm{NaOCl}$ for about one hour to remove any organic material and dissolved in doubly distilled 0.1 M acetic acid (Asahara et al., 1995) by placement in an ultrasonic bath for $0.5 \mathrm{~h}$. Samples were then allowed to stand for $\sim 12 \mathrm{~h}$ before decanting the acid. $\mathrm{An}^{84} \mathrm{Sr}$ spike was added to all water and some carbonate samples for the purpose of obtaining 


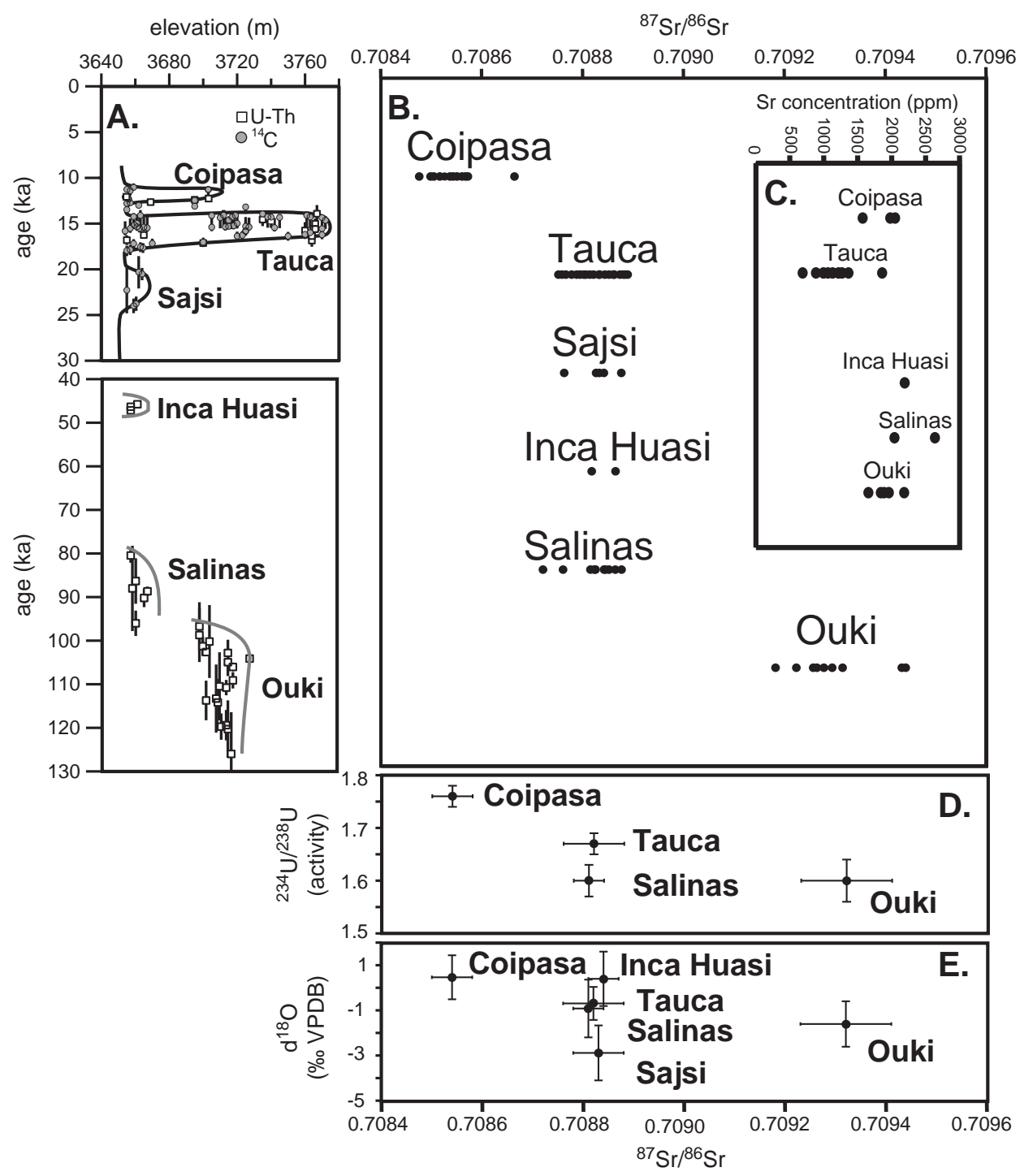

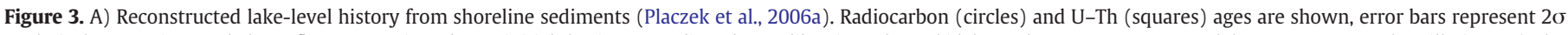

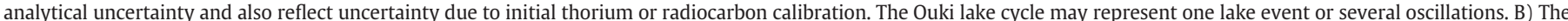

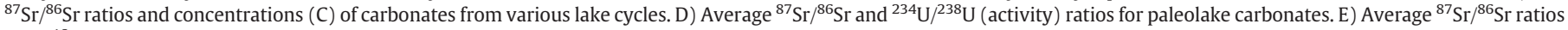
and $\delta^{18} \mathrm{O}$ values for paleolake carbonates.

strontium concentration data, and ${ }^{87} \mathrm{Sr} /{ }^{86} \mathrm{Sr}$ ratios of spiked samples are corrected for spike composition. Strontium was separated with Eichrom strontium-specific resin, and ${ }^{87} \mathrm{Sr} /{ }^{86} \mathrm{Sr}$ ratios were measured on a Micromass Sector 54 thermal-ionization mass spectrometer. The ${ }^{87} \mathrm{Sr} /{ }^{86} \mathrm{Sr}$ ratio was normalized to 0.1194 and analyses of the NBS987 standard run on each 20-sample turret yielded a mean ratio of $0.71026 \pm 0.00002(\mathrm{n}=12)$.

Water isotopic results are reported using standard $\delta$-per mil notation and were processed at the Laboratory of Isotope Geochemistry, University of Arizona. Water samples were prepared using the $\mathrm{CO}_{2}$ equilibration method on a Kiel III attached to a Finnigan Delta Plus mass spectrometer. The values were corrected based on internal lab standards, which are calibrated through VSMOW and SLAP. The analytical precision is $0.08 \%$ and $0.1 \%$, respectively $(1 \sigma)$. Carbonate samples were treated with $2 \% \mathrm{H}_{2} \mathrm{O}_{2}$ for $2 \mathrm{~h}$ to remove organic matter and converted to gas using $100 \%$ phosphoric acid on a Keil III attached to a Finnigan Delta $\mathrm{S}$ mass spectrometer. These values were corrected based on internal lab standards, which are calibrated through VPDB. The analytical precision is $0.05 \%$ 。 $(1 \sigma)$.

The initial ${ }^{234} \mathrm{U} /{ }^{238} \mathrm{U}$ ratio (expressed throughout this paper as activity ratios) of paleolake carbonates is calculated from the data set of Placzek et al. (2006a). These initial ${ }^{234} U /{ }^{238} U$ ratios are from $U-$ ${ }^{230} \mathrm{Th}$ dates with small corrections for initial ${ }^{230} \mathrm{Th}$; reported uncertainties include propagated $2 \sigma$ envelopes on isotope ratios, decay constants, as well as error on the assumed initial ${ }^{230} \mathrm{Th} /{ }^{232} \mathrm{Th}$ ratio. Carbonates were dissolved in $2 \mathrm{M} \mathrm{HNO}_{3}$ and spiked with mixed ${ }^{233} \mathrm{U}^{229} \mathrm{Th}$. Uranium and Th were co-precipitated with $\mathrm{FeOH}_{3}$, separated by anion exchange, and measured on a Micromass Sector 54 thermal ionization mass spectrometer in the Department of Geosciences at the University of Arizona. Details of our analytical procedures are fully discussed in Placzek et al. (2006b). Decay constants are after Cheng et al. (2000).

\section{Results}

\section{Modern waters}

The ${ }^{87} \mathrm{Sr} /{ }^{86} \mathrm{Sr}$ ratios of modern waters on the Bolivian Altiplano range from 0.70548 to 0.72553 and strontium concentrations range between 0.03 and 13.8 ppm (Table 1 ; Fig. 4 ). Catchments that drain the WC generally have ratios less than 0.708 . In contrast, only catchments that drain the EC have ${ }^{87} \mathrm{Sr} /{ }^{86} \mathrm{Sr}$ ratios above 0.710 (Table 1; Fig. 4). 
Table 1

Isotopic data from Altiplano waters.

\begin{tabular}{|c|c|c|c|c|c|c|c|c|}
\hline Catchment \#, basin & River & ${ }^{87} \mathrm{Sr} /{ }^{86} \mathrm{Sr}( \pm 1 \mathrm{SE})$ & Sr ppm $( \pm 1 \mathrm{SE})$ & Area $\left(\mathrm{km}^{2}\right)$ & Rain shadow & $\delta^{18} O^{a}$ & $\delta^{2} \mathrm{H}^{\mathrm{a}}$ & Geology \\
\hline $1^{\mathrm{b}}$, Titicaca & Río Ramis & $0.70870 \pm 1$ & 0.675 & 4210 & No & 0.0 & 0.0 & Pliocene through Quaternary volcanics with minor Jurassic through Tertiary sediments \\
\hline $2^{\mathrm{b}}$, Titicaca & Río Huancané & $0.71028 \pm 1$ & 0.709 & 4240 & Yes & & & Cretaceous through Devonian sediments \\
\hline $3^{\mathrm{b}}$, Titicaca & Río Suches & $0.71200 \pm 05$ & 0.135 & 3370 & Yes & & & Cretaceous through Devonian sediments \\
\hline $4^{\mathrm{b}}$, Titicaca & Río Huaychoc & $0.70973 \pm 1$ & 0.301 & 1020 & Yes & & & Cretaceous through Devonian sediments \\
\hline $5^{\mathrm{b}}$, Titicaca & Río Coata & $0.70718 \pm 1$ & 1.051 & 1550 & No & & & Pliocene through Quaternary volcanics with minor Jurassic through Tertiary sediments \\
\hline $6^{\mathrm{b}}$, Titicaca & Río lipa $^{c}$ & $0.70746 \pm 1$ & 0.777 & 1280 & No & & & Pliocene through Quaternary volcanics with minor Jurassic through Tertiary sediments \\
\hline $7^{\mathrm{b}}$, Titicaca & Río Ilave & $0.70721 \pm 1$ & 0.301 & 14,410 & No & & & Pliocene through Quaternary volcanics with minor Jurassic through Tertiary sediments \\
\hline $8^{\mathrm{b}}$, Titicaca & Río Keka & $0.72196 \pm 1$ & 0.029 & 1680 & Yes & & & Devonian sediments and Neogene plutons and sediments \\
\hline 9, Titicaca & Río Catari & Río Keka values & Río Keka values & 4700 & Yes & & & Devonian plutons and Neogene sediments \\
\hline 10, Titicaca & Río Tiahuanacu & Río Iipa values & Río Iipa values & 840 & No & & & Neogene plutons and sediments \\
\hline 11, Poópo & Río Jacha Jahuira & $0.70948 \pm 1$ & 0.946 & 6370 & No & -4.0 & -65.5 & Paleogene to Neogene sediments with minor Proterozoic crystalline rock \\
\hline $12^{\mathrm{b}}$, Titicaca & Río Tujso Johuiro & Río Jacha Jahuira values & Río Jacha Jahuira values & 3920 & No & & & Paleogene to Neogene sediments and volcanics \\
\hline 13 $3^{\mathrm{b}}$, Poópo & Río Mauri & $0.70735 \pm 1$ & 0.199 & 12,110 & No & & & Neogene volcanics and plutons \\
\hline 14, Poópo & Well water & $0.70968 \pm 1$ & 0.199 & 16,320 & No & & & Paleogene to Neogene sediments \\
\hline 15, Poópo & $\mathrm{n} / \mathrm{a}$ & Catchment 14 values & Catchment 14 values & 10,400 & Yes & & & Silurian sediments and Neogene tuffs \\
\hline 16 , Poópo & Río Caranguila & $0.70976 \pm 1$ & 0.769 & 7400 & No & & & Silurian sediments and Neogene tuffs \\
\hline 17, Coipasa & Río Lauca & $0.70715 \pm 1$ & $0.684 \pm 0.005$ & 10,440 & No & -8.8 & -91.1 & Neogene through Quaternary volcanics and tuffs \\
\hline 18b, Poópo & Río Poópo & $0.72412 \pm 1$ & 1.113 & 8500 & Yes & & & Neogene through Quaternary volcanics and tuffs \\
\hline 19, Coipasa & Río Barras & $0.72412 \pm 2$ & $0.380 \pm 0.003$ & 5530 & No & -9.4 & -94.8 & Silurian sediments and Neogene tuffs \\
\hline 20, Coipasa & Seep & $0.70679 \pm 2$ & $1.193 \pm 0.008$ & 8880 & No & -8.5 & -92.0 & Paleogene to Neogene sediments and Cretaceous sediments \\
\hline 21, Coipasa/Uyuni & Unnamed river & $0.70963 \pm 1$ & $13.79 \pm 0.18$ & 7470 & Yes & -5.4 & -78.6 & Neogene through Quaternary volcanics and tuffs \\
\hline 22, Poópo & Río Sevaruyo & $0.71637 \pm 2$ & 0.097 & 3170 & No & & & Neogene tuffs and Cretaceous volcanics \\
\hline 23, Poópo & Río Quillacas & $0.71591 \pm 1$ & $0.321 \pm 0.002$ & 1410 & Yes & 3.0 & -48.8 & Neogene tuff \\
\hline 24, Uyuni & Río Sajsi & $0.70946 \pm 1$ & $2.40 \pm 0.06$ & 3820 & No & & & Neogene tuff and Cretaceous sediments \\
\hline 25, Uyuni & Río Kolcha "K" & $0.70678 \pm 7$ & $1.23 \pm 0.02$ & 6790 & No & -7.6 & -86.1 & Cretaceous sediments; Paleogene through Neogene sediments, plutonics and volcanics \\
\hline 26, Uyuni & Río Puca Mayu & $0.71176 \pm 2$ & $13.8 \pm 0.6$ & 10,490 & Yes & 0.2 & -18.0 & Silurian sediments; Paleogene to Neogene sediments and tuffs \\
\hline 27 , Uyuni & Río Grande & $0.70952 \pm 1$ & $2.76 \pm 0.02$ & 30,584 & No & $-4.1 ;-1.4^{\mathrm{d}}$ & $-63.5 ;-53.1^{d}$ & $\begin{array}{l}\text { Neogene tuffs and volcanics; Paleogene to Neogene sediments, with minor Ordivician } \\
\text { and Silurian sediments }\end{array}$ \\
\hline Catchment \#, basin & Sample type & ${ }^{87} \mathrm{Sr} /{ }^{86} \mathrm{Sr}( \pm 1 \mathrm{SE})$ & $\operatorname{Sr} \operatorname{ppm}( \pm 1 \mathrm{SE})$ & \multicolumn{2}{|c|}{ Latitude; longitude } & & & \\
\hline Uyuni & Uyuni water & $0.70894 \pm 1$ & $30.7 \pm 0.5$ & \multirow{2}{*}{\multicolumn{2}{|c|}{$\begin{array}{l}20^{\circ} 07^{\prime} \mathrm{S} ; 68^{\circ} 14^{\prime} \mathrm{W} \\
20^{\circ} 18^{\prime} \mathrm{S} ; 67^{\circ} 22^{\prime} \mathrm{W}\end{array}$}} & -3.3 & -36.0 & \\
\hline Uyuni & Uyuni salt & $0.70885 \pm 0.01$ & $2637 \pm 0.93$ & & & & & \\
\hline Uyuni $^{\mathrm{b}}$ & Uyuni water & $0.70878 \pm 1$ & 9.90 & \multicolumn{2}{|c|}{$20^{\circ} 34^{\prime} \mathrm{S} ; 67^{\circ} 33^{\prime} \mathrm{W}$} & 4.1 & -4.0 & \\
\hline Uyuni & Spring in Uyuni & $0.70874 \pm 1$ & $4.5 \pm 0.1$ & \multicolumn{2}{|c|}{$20^{\circ} 18^{\prime} \mathrm{S} ; 67^{\circ} 22^{\prime} \mathrm{W}$} & & & \\
\hline Coipasa & Coipasa water & $0.70915 \pm 1$ & $49.8 \pm 1.5$ & \multicolumn{2}{|c|}{$19^{\circ} 32^{\prime} \mathrm{S} ; 67^{\circ} 55^{\prime} \mathrm{W}$} & 2.0 & -30.0 & \\
\hline Coipasa & Coipasa salt & $0.70827 \pm 1$ & $67.8 \pm 0.7$ & \multicolumn{2}{|c|}{$19^{\circ} 32^{\prime} \mathrm{S} ; 67^{\circ} 55^{\prime} \mathrm{W}$} & & & \\
\hline Poópo & Poópo water & $0.70938 \pm 1$ & $9.45 \pm 0.05$ & \multicolumn{2}{|c|}{$18^{\circ} 30^{\prime} \mathrm{S} ; 67^{\circ} 15^{\prime} \mathrm{W}$} & 22.5 & 36.4 & \\
\hline Poópo & Poópo water & $0.71404 \pm 1$ & 1.32 & \multicolumn{2}{|c|}{$18^{\circ} 23^{\prime} \mathrm{S} ; 66^{\circ} 57^{\prime} \mathrm{W}$} & & & \\
\hline Titicacab & Modern Lago Grande & $0.70822 \pm 0.6$ & 1.15 & \multicolumn{2}{|c|}{$18^{\circ} 30^{\prime} \mathrm{S} ; 67^{\circ} 15^{\prime} \mathrm{W}$} & & & \\
\hline Titicacab & Pleistocene overflow & 0.70830 & & & & & & \\
\hline Poópo & Río Desaguadero 1 & $0.70885 \pm 1$ & $1.90 \pm 0.01$ & \multicolumn{2}{|c|}{$18^{\circ} 13^{\prime} \mathrm{S} ; 67^{\circ} 09^{\prime} \mathrm{W}$} & 6.4 & -30.5 & \\
\hline Poópo & Río Desaguadero 2 & $0.70874 \pm 1$ & & \multicolumn{2}{|c|}{$18^{\circ} 05^{\prime} \mathrm{S} ; 67^{\circ} 09^{\prime} \mathrm{W}$} & -0.2 & -49.1 & \\
\hline Poópo & Río Desaguadero 3 & $0.70916 \pm 1$ & $1.40 \pm 0.02$ & \multirow{2}{*}{\multicolumn{2}{|c|}{$\begin{array}{l}18^{\circ} 01^{\prime} \mathrm{S} ; 67^{\circ} 09^{\prime} \mathrm{W} \\
19^{\circ} 50^{\prime} \mathrm{S} ; 67^{\circ} 27^{\prime} \mathrm{W}\end{array}$}} & 3.0 & -48.2 & \\
\hline 24 & Río Sajsi well & $0.70792 \pm 1$ & $10.5 \pm 0.1$ & & & -11.5 & -102.7 & \\
\hline 25 & Empexa spring & $0.70548 \pm 1$ & $1.96 \pm 0.02$ & \multicolumn{2}{|c|}{$20^{\circ} 32^{\prime} \mathrm{S} ; 68^{\circ} 26^{\prime} \mathrm{W}$} & -10.2 & -90.2 & \\
\hline 21 & Spring & $0.70620 \pm 1$ & $2.58 \pm 0.02$ & \multicolumn{2}{|c|}{$\begin{array}{l}19^{\circ} 19^{\prime} \mathrm{S} ; 68^{\circ} 22^{\prime} \mathrm{W} \\
\end{array}$} & -8.9 & -96.4 & \\
\hline 18 & Pazña hot springs & $0.72553 \pm 3$ & $2.87 \pm 0.5$ & \multicolumn{2}{|c|}{$18^{\circ} 35^{\prime} \mathrm{S} ; 66^{\circ} 56^{\prime} \mathrm{W}$} & -8.7 & -89.6 & \\
\hline
\end{tabular}

a Typical standard error is $0.1 \%$.

Data from Grove et al. (2003).

Wet season data, all other data is from the dry season.

${ }^{d}$ Two stable isotope samples collected (different seasons). 


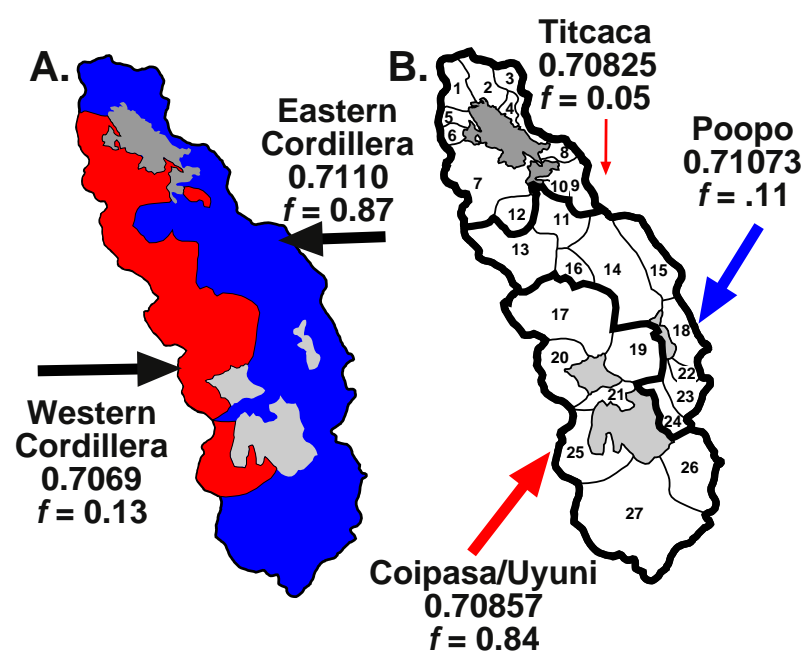

Figure 4. A) The distribution of ${ }^{87} \mathrm{Sr} /{ }^{86} \mathrm{Sr}$ ratios of modern catchments on the Bolivian Altiplano based on river and creek sampling. Gray denotes modern lakes and salars. Black represents catchments with ratios $<0.709$ and white represents ratios $>0.709$. Ratios of ${ }^{87} \mathrm{Sr} /{ }^{86} \mathrm{Sr}$ and fractional $\mathrm{Sr}$ contributions $(f)$ for the EC and WC are given (see text for computation of $f$ ). B) Catchments and sub-basins of the Bolivian Altiplano; individual catchment numbers correspond to Table 1 . Ratios of ${ }^{87} \mathrm{Sr} /{ }^{86} \mathrm{Sr}$ and fractional Sr contributions are given for Lake Titicaca, the Poópo basin, and the Coipasa/Uyuni basin.

Some groundwater ${ }^{87} \mathrm{Sr} /{ }^{86} \mathrm{Sr}$ ratios were measured, and ratios for groundwater are similar to those of local surface waters (Table 1 ).

The $\delta^{18} \mathrm{O}$ values of modern water on the Altiplano vary between -11.5 and $-22.0 \%$, with $\delta^{2} \mathrm{H}$ values between -102.7 and $36.4 \%$. The $\delta^{18} \mathrm{O}$ value of modern lake Titicaca is -3.8 to $-4.6 \%$ (Cross et al., 2001). Measured values for modern waters from the Poópo, Coipasa, and Uyuni basins are between -11.5 and $-22.0 \%$ (Table 1; Fig. 5). Modern rainfall values average about $-17.5 \%$ (Cross et al., 2001).

\section{Paleolake carbonates}

Paleolake carbonates from the Bolivian Altiplano have variable ${ }^{87} \mathrm{Sr} /{ }^{86} \mathrm{Sr}$ and ${ }^{234} \mathrm{U} /{ }^{238} \mathrm{U}$ ratios, but $\delta^{18} \mathrm{O}$ values for all lake cycles are similar (Fig. 3; Table 3). The ${ }^{87} \mathrm{Sr} /{ }^{86} \mathrm{Sr}$ ratios range between 0.70848 and 0.70944 and strontium concentrations are between 690 and 2640 ppm (Fig. 3; Table 2). Shoreline carbonates from the southern Altiplano also have variable initial ${ }^{234} \mathrm{U} /{ }^{238} \mathrm{U}$ ratios between 1.6 and 1.76 (Fig. 3D). The average $\delta^{18} \mathrm{O}$ values for all lake cycles are similar, ranging between -2.9 and $0.5 \%$; the scatter within individual lake cycles produces overlapping $\delta^{18} \mathrm{O}$ values for all lake cycles (Fig. 3E; Supplementary data).

Carbonates from the Coipasa lake cycle have the lowest ${ }^{87} \mathrm{Sr} /{ }^{86} \mathrm{Sr}$ ratios $(0.70854 \pm 0.00004)$ and the highest initial ${ }^{234} \mathrm{U} /{ }^{238} \mathrm{U}(1.79 \pm$

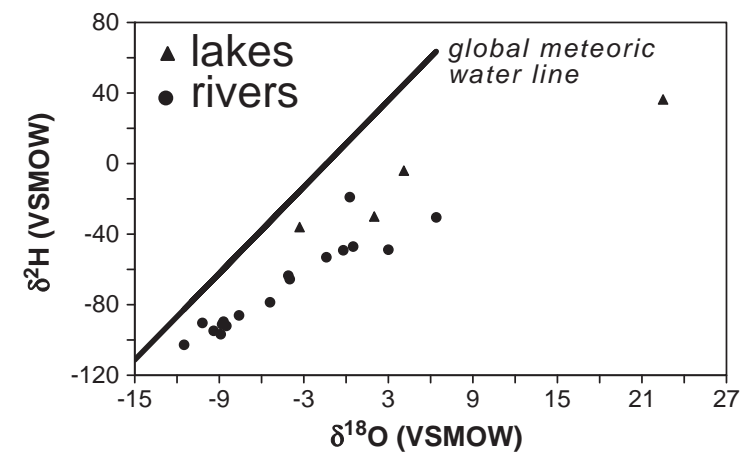

Figure 5. Values of $\delta^{18} \mathrm{O}$ and $\delta^{2} \mathrm{H}$ from modern rivers (circles) and lakes (triangles) from the southern Altiplano (Table 2). The global meteoric water line (GMWL) is shown; samples show variable degrees of evaporative enrichment.
0.06) (Table 2); the average $\delta^{18} \mathrm{O}$ for the Coipasa lake cycle is $0.4 \pm 1.0$. The population of carbonates with ${ }^{87} \mathrm{Sr} /{ }^{86} \mathrm{Sr}$ ratios below 0.7087 includes six undated samples with field associations indicative of formation during the Coipasa lake cycle. One carbonate, whose age and depositional context indicate that it formed during the regression of the Coipasa lake cycle, has a somewhat higher ${ }^{87} \mathrm{Sr} /{ }^{86} \mathrm{Sr}$ ratio (0.70867) and was collected along a perennial wash where mixing with local river water may have occurred at low lake levels.

The ${ }^{87} \mathrm{Sr} /{ }^{86} \mathrm{Sr}$ ratio of carbonates of the Tauca, Salinas, Inca Huasi, and Sajsi lake cycles are between 0.70872 and 0.70895 , and ${ }^{234} U /{ }^{238} U$ ratios range between 1.61 and 1.69 (Table 2); the average $\delta^{18} \mathrm{O}$ for the Tauca, Sajsi, Inca Huasi, and Salinas lake cycles are: $-0.95 \% \_ \pm 1.3$; $-2.9 \%$ $\pm 1.2 ;+0.4 \%$ $\pm 1.2 ;-0.7 \%$ \pm 0.7 , respectively. Ratios of ${ }^{87} \mathrm{Sr} /$ ${ }^{86} \mathrm{Sr}$ are $\sim 0.7088$ for all samples from the Tauca highstand at diverse locations around the basins (Table 2). Carbonates that mark the transgression and regression of the Tauca lake cycle to elevations lower than the Laca hydrologic divide $\left(3700 \mathrm{~m}\right.$ ) also have ${ }^{87} \mathrm{Sr} /{ }^{86} \mathrm{Sr}$ ratios of $\sim 0.7088$ (Table 2). Carbonates from the Salinas lake cycle (95 to $80 \mathrm{ka}$ ) have ${ }^{87} \mathrm{Sr} /{ }^{86} \mathrm{Sr}$ ratios between 0.70872 and 0.70885 . Carbonates attributed to the Inca Huasi ( $46 \mathrm{ka}$ ) and Sajsi (24$20.5 \mathrm{ka}$ ) lake cycles yielded ${ }^{87} \mathrm{Sr} /{ }^{86} \mathrm{Sr}$ ratios between 0.70876 and 0.70888 , but are measured at only one (Sajsi) or two (Inca Huasi) locations.

Carbonates from the Ouki deep lake cycle (120-95 ka) have ${ }^{87} \mathrm{Sr} /$ ${ }^{86} \mathrm{Sr}$ ratios ranging from 0.70918 to 0.70944 and ${ }^{234} \mathrm{U} /{ }^{238} \mathrm{U}$ ratios of $1.60 \pm 0.04$. The average $\delta^{18} \mathrm{O}$ value for the Ouki lake cycle is $-1.6 \% \pm$ 1.0. These are the highest ${ }^{87} \mathrm{Sr} /{ }^{86} \mathrm{Sr}$ ratios for carbonates of any lake cycle. Strontium concentrations for this lake cycle are also relatively high, between 1657 and 1958 ppm (Fig. 3C).

\section{Modeling the modern lake system}

${ }^{87} \mathrm{Sr} /{ }^{86} \mathrm{Sr}$

We constructed a mass balance model of $\mathrm{Sr}$ inputs to the modern lake that assists in understanding the ${ }^{87} \mathrm{Sr} /{ }^{86} \mathrm{Sr}$ ratio of the paleolakes. Much of this model has been developed in previous studies (Grove et al., 2003; Coudrain et al., 2002). In this study we add a substantial body of new evidence from the southern basins. Modeling of the lake ${ }^{87} \mathrm{Sr} /{ }^{86} \mathrm{Sr}_{\text {lake }}$ ratio was estimated by:

$$
\begin{aligned}
& { }^{87} \mathrm{Sr} /{ }^{86} \mathrm{Sr}_{\text {lake }}=f_{\text {Sr River1 }}\left({ }^{87} \mathrm{Sr} /{ }^{86} \mathrm{Sr}_{\text {River1 }}\right)+f_{\text {Sr River2 }}\left({ }^{87} \mathrm{Sr} /{ }^{86} \mathrm{Sr} \text { River2 }\right) \ldots \\
& +f_{\text {Sr groundwater }}\left({ }^{87} \mathrm{Sr} /{ }^{86} \mathrm{Sr}_{\text {groundwater }}\right)+f_{\text {Sr Tticaca }}\left({ }^{87} \mathrm{Sr} /{ }^{86} \mathrm{Sr} \text { Titicaca }\right) \text {. }
\end{aligned}
$$

Here $f_{\text {Sr River } 1 \text {, River 2, etc. }}=$ the fraction of $\mathrm{Sr}$ from each of the major

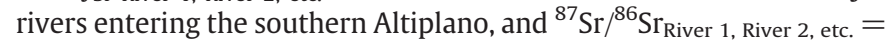
the ${ }^{87} \mathrm{Sr} /{ }^{86} \mathrm{Sr}$ ratio for each of the major rivers. Here, we assume that the $\mathrm{Sr}$ contribution from direct rainfall onto the lake surface is negligible. In contrast, the groundwater term can make significant contributions to Sr budgets (e.g. Hart et al., 2004) and can be difficult to establish. In most cases, groundwater contributes primarily to streams rather than flowing into lakes, allowing accurate Sr budget models without direct consideration of this term (e.g. Pretti and Stewart, 2002). This assumption was proven valid on the Altiplano by Grove et al. (2003), who successfully reconstructed the modern ${ }^{87} \mathrm{Sr} /{ }^{86} \mathrm{Sr}$ for Lake Titicaca without the groundwater term, and we adopt this simplification in our modern and paleolake models.

Mass balance models typically describe the Sr fractional contribution $(f)$ of individual watershed (River 1) as:

$$
f_{\text {river1 }}=\frac{\left(\text { concSr }_{\text {river1 } 1}\right) \times\left(\text { discharge }_{\text {river1 } 1}\right)}{\sum_{\text {river1 } 1}^{\text {river }}\left(\operatorname{concSr}_{\text {river1 } 1,2,3, \ldots x}\right) \times\left(\text { discharg }_{\text {river } 1,2,3, \ldots x}\right)}
$$


Table 2

Isotopic data from carbonates.

\begin{tabular}{|c|c|c|c|c|c|}
\hline Sample/lake cycle & ${ }^{87} \mathrm{Sr} /{ }^{86} \mathrm{Sr}( \pm 1 \mathrm{SE})$ & Sr ppm $( \pm 1 \mathrm{SE})$ & Sample material & Sample location (latitude; longitude; site $\#^{\mathrm{a}}$ ) & $\delta^{18} \mathrm{O}^{\mathrm{b}}(\%)$ \\
\hline \multicolumn{6}{|l|}{ Coipasa } \\
\hline$S-8-2$ & $0.70856 \pm 1$ & $1978 \pm 2$ & Tufa & $19^{\circ} 50^{\prime} \mathrm{S} ; 67^{\circ} 09^{\prime} \mathrm{W} ; 14$ & -0.5 \\
\hline S-10-3 & $0.70856 \pm 1$ & & Tufa & $19^{\circ} 50^{\prime} \mathrm{S} ; 67^{\circ} 09^{\prime} \mathrm{W} ; 14$ & 0.0 \\
\hline U-31-3 & $0.70852 \pm 1$ & & Tufa & $20^{\circ} 14^{\prime} \mathrm{S} ; 67^{\circ} 38^{\prime} \mathrm{W} ; 23$ & $1.7 ; 0.4$ \\
\hline U-31-4 & $0.70852 \pm 1$ & & Tufa & $20^{\circ} 14^{\prime} \mathrm{S} ; 67^{\circ} 38^{\prime} \mathrm{W} ; 23$ & $2.1 ; 1.4$ \\
\hline U-23a-3 & $0.70852 \pm 1$ & & Tufa & $20^{\circ} 57^{\prime} \mathrm{S} ; 67^{\circ} 02^{\prime} \mathrm{W}$ & 0.5 \\
\hline$U-23 a-4$ & $0.70850 \pm 1$ & & Tufa & $20^{\circ} 57^{\prime} \mathrm{S} ; 67^{\circ} 02^{\prime} \mathrm{W}$ & 0.1 \\
\hline C-14-1 & $0.70853 \pm 1$ & & Tufa & $19^{\circ} 07^{\prime} \mathrm{S} ; 67^{\circ} 47^{\prime} \mathrm{W}$ & \\
\hline $\mathrm{U}-32-3$ & $0.70854 \pm 1$ & & Tufa & $20^{\circ} 14^{\prime} \mathrm{S} ; 67^{\circ} 37^{\prime} \mathrm{W}$ & \\
\hline U-9-6 & $0.70855 \pm 1$ & $1578 \pm 0.5$ & Tufa & $20^{\circ} 36^{\prime} \mathrm{S} ; 67^{\circ} 37^{\prime} \mathrm{W} ; 25$ & $1.1 ; 0.1 ;-1.2$ \\
\hline B-17 & $0.70854 \pm 1$ & $1572 \pm 1$ & Tufa & $19^{\circ} 51^{\prime} \mathrm{S} ; 67^{\circ} 33^{\prime} \mathrm{W} ; 15$ & $0.0 ; 0.3$ \\
\hline U-27b-6 & $0.70855 \pm 1$ & $2050 \pm 2$ & Tufa & $20^{\circ} 09^{\prime} \mathrm{S} ; 67^{\circ} 49^{\prime} \mathrm{W} ; 19$ & \\
\hline U-27b-8 & $0.70853 \pm 1$ & & Tufa & $20^{\circ} 09^{\prime} \mathrm{S} ; 67^{\circ} 49^{\prime} \mathrm{W} ; 19$ & \\
\hline U-27a-2 & $0.70853 \pm 1$ & & Tufa & $20^{\circ} 49^{\prime} \mathrm{S} ; 67^{\circ} 16^{\prime} \mathrm{W}$ & \\
\hline$U-27 a-3 e x$ & $0.70857 \pm 1$ & & Tufa & $20^{\circ} 49^{\prime} \mathrm{S} ; 67^{\circ} 16^{\prime} \mathrm{W}$ & \\
\hline$U-27 a-6$ & $0.70851 \pm 1$ & & Tufa & $20^{\circ} 49^{\prime} \mathrm{S} ; 67^{\circ} 16^{\prime} \mathrm{W}$ & $1.6 ; 0.3$ \\
\hline $\mathrm{U}-10$ & $0.70850 \pm 1$ & $2300 \pm 0.5$ & Tufa & $20^{\circ} 49^{\prime} \mathrm{S} ; 67^{\circ} 09^{\prime} \mathrm{W} ; 30$ & $-0.3 ; 1.8$ \\
\hline$S-6-3^{c}$ & $0.70867 \pm 1$ & & Tufa & $19^{\circ} 50^{\prime} \mathrm{S} ; 67^{\circ} 09^{\prime} \mathrm{W} ; 14$ & -1.2 \\
\hline Average & $0.70854 \pm 4$ & $1990 \pm 320$ & & & $0.4 \pm 1.00$ \\
\hline \multicolumn{6}{|l|}{ Tauca transgression } \\
\hline$U-29 a-1$ & $0.70887 \pm 1$ & $1130 \pm 1$ & Ooids & $20^{\circ} 07^{\prime} \mathrm{S} ; 67^{\circ} 02^{\prime} \mathrm{W} ; 18$ & 1.3 \\
\hline U-27b-2 & $0.70881 \pm 1$ & $1859 \pm 2$ & Tufa & 0.7 & 0.7 \\
\hline S-17-4 & $0.70884 \pm 2$ & & Tufa & $19^{\circ} 50^{\prime} \mathrm{S} ; 67^{\circ} 09^{\prime} \mathrm{W} ; 14$ & \\
\hline S-6-1 & $0.70887 \pm 1$ & & Tufa & $19^{\circ} 50^{\prime} \mathrm{S} ; 67^{\circ} 09^{\prime} \mathrm{W} ; 14$ & \\
\hline Average & $0.70885 \pm 3$ & & & & $1.0 \pm 0.3$ \\
\hline \multicolumn{6}{|l|}{ Tauca } \\
\hline$S-17-5$ & $0.70884 \pm 2$ & & Tufa & $19^{\circ} 50^{\prime} \mathrm{S} ; 67^{\circ} 09^{\prime} \mathrm{W} ; 14$ & -1.2 \\
\hline S-17-6 & $0.70883 \pm 1$ & & Tufa & $19^{\circ} 50^{\prime} \mathrm{S} ; 67^{\circ} 09^{\prime} \mathrm{W} ; 14$ & -0.5 \\
\hline RG2-1 & $0.70889 \pm 1$ & & Tufa & $20^{\circ} 47^{\prime} \mathrm{S} ; 67^{\circ} 06^{\prime} \mathrm{W}$ & -1.1 \\
\hline RG2-3 & & & Tufa & $20^{\circ} 47^{\prime} \mathrm{S} ; 67^{\circ} 06^{\prime} \mathrm{W}$ & 0.6 \\
\hline CL-2 & $0.70877 \pm 1$ & & Tufa & Ascotan, Chile & 0.2 \\
\hline U-23a-2 & $0.70885 \pm 1$ & & Tufa & $19^{\circ} 41^{\prime} \mathrm{S} ; 67^{\circ} 39^{\prime} \mathrm{W}$ & \\
\hline U-31-2 & $0.70881 \pm 1$ & & Tufa & $20^{\circ} 14^{\prime} \mathrm{S} ; 67^{\circ} 38^{\prime} \mathrm{W} ; 23$ & \\
\hline U-26-5 & $0.70882 \pm 1$ & & Tufa & $20^{\circ} 48^{\prime} \mathrm{S} ; 67^{\circ} 39^{\prime} \mathrm{W} ; 24$ & \\
\hline U-23a-1 & $0.70885 \pm 1$ & & Tufa & $20^{\circ} 57^{\prime} \mathrm{S} ; 67^{\circ} 02^{\prime} \mathrm{W}$ & \\
\hline C-1-1 & $0.70880 \pm 1$ & $1139 \pm 0.5$ & Tufa & $19^{\circ} 37^{\prime} \mathrm{S} ; 68^{\circ} 06^{\prime} \mathrm{W}$ & -2.5 \\
\hline$C-1-2$ & $0.70880 \pm 6$ & $1035 \pm 0.5$ & Tufa & $19^{\circ} 37^{\prime} \mathrm{S} ; 68^{\circ} 06^{\prime} \mathrm{W}$ & -2.2 \\
\hline$C-1-3$ & $0.70880 \pm 1$ & $1268 \pm 0.5$ & Tufa & $19^{\circ} 37^{\prime} \mathrm{S} ; 68^{\circ} 06^{\prime} \mathrm{W}$ & -1.5 \\
\hline$C-1-4$ & $0.70885 \pm 1$ & $1205 \pm 0.5$ & Tufa & $19^{\circ} 37^{\prime} \mathrm{S} ; 68^{\circ} 06^{\prime} \mathrm{W}$ & -0.8 \\
\hline U-5-1 & $0.70881 \pm 1$ & $1045 \pm 0.5$ & Tufa & $20^{\circ} 36^{\prime} \mathrm{S} ; 67^{\circ} 35^{\prime} \mathrm{W} ; 25$ & $-1.6 ;-2.0$ \\
\hline$U-5-2$ & $0.70877 \pm 1$ & $1120 \pm 0.5$ & Tufa & $20^{\circ} 36^{\prime} \mathrm{S} ; 67^{\circ} 35^{\prime} \mathrm{W} ; 25$ & $-1.9 ;-2.4$ \\
\hline$U-5-3$ & $0.70885 \pm 1$ & $995 \pm 0.5$ & Tufa & $20^{\circ} 36^{\prime} \mathrm{S} ; 67^{\circ} 35^{\prime} \mathrm{W} ; 25$ & \\
\hline U-9-2 & $0.70879 \pm 1$ & $1161 \pm 0.5$ & Tufa & $19^{\circ} 50^{\prime} \mathrm{S} ; 67^{\circ} 33^{\prime} \mathrm{W} ; 15$ & $-0.5 ;-0.9$ \\
\hline U-9-1 & & & Tufa & $19^{\circ} 50^{\prime} \mathrm{S} ; 67^{\circ} 33^{\prime} \mathrm{W} ; 15$ & -2.4 \\
\hline U-9-3 & & & Tufa & $19^{\circ} 50^{\prime} \mathrm{S} ; 67^{\circ} 33^{\prime} \mathrm{W} ; 15$ & -1.8 \\
\hline U-9-4 & & & Tufa & $19^{\circ} 50^{\prime} \mathrm{S} ; 67^{\circ} 33^{\prime} \mathrm{W} ; 15$ & -1.4 \\
\hline U-9-5 & & & Tufa & $19^{\circ} 50^{\prime} \mathrm{S} ; 67^{\circ} 33^{\prime} \mathrm{W} ; 15$ & -1.0 \\
\hline U-7-1 & $0.70881 \pm 1$ & $1215 \pm 0.5$ & Tufa & $20^{\circ} 43^{\prime} \mathrm{S} ; 67^{\circ} 59^{\prime} \mathrm{W} ; 29$ & -1.5 \\
\hline $\mathrm{U}-7-2$ & $0.70884 \pm 1$ & $1363 \pm 0.5$ & Tufa & $20^{\circ} 43^{\prime} \mathrm{S} ; 67^{\circ} 59^{\prime} \mathrm{W} ; 29$ & \\
\hline U-11-2 & $0.70883 \pm 1$ & $1060 \pm 0.5$ & Tufa & $20^{\circ} 49^{\prime} \mathrm{S} ; 67^{\circ} 08^{\prime} \mathrm{W}$ & -1.9 \\
\hline P-3-1 & $0.70888 \pm 1$ & $890 \pm 0.5$ & Tufa & $18^{\circ} 34^{\prime} \mathrm{S} ; 66^{\circ} 57^{\prime} \mathrm{W}$ & $-2.1 ;-2.9$ \\
\hline P-3-11 & $0.70884 \pm 2$ & $883 \pm 1$ & Tufa & $19^{\circ} 21^{\prime} \mathrm{S} ; 67^{\circ} 13^{\prime} \mathrm{W}$ & -2.3 \\
\hline B-8 & $0.70881 \pm 1$ & $1154 \pm 0.5$ & Tufa & $19^{\circ} 51^{\prime} \mathrm{S} ; 67^{\circ} 33^{\prime} \mathrm{W} ; 15$ & -1.1 \\
\hline$U-27 a-3 e x$ & $0.70879 \pm 1$ & & Tufa & $20^{\circ} 09^{\prime} \mathrm{S} ; 67^{\circ} 49^{\prime} \mathrm{W} ; 19$ & \\
\hline$U-27 a-5$ & $0.70875 \pm 3$ & & Tufa & $20^{\circ} 09^{\prime} \mathrm{S} ; 67^{\circ} 49^{\prime} \mathrm{W} ; 19$ & 1.6 \\
\hline$U-27 a-4$ & $0.70881 \pm 2$ & & Tufa & $20^{\circ} 09^{\prime} \mathrm{S} ; 67^{\circ} 49^{\prime} \mathrm{W} ; 19$ & \\
\hline U-27a-3in & $0.70883 \pm 1$ & & Tufa & $20^{\circ} 09^{\prime} \mathrm{S} ; 67^{\circ} 49^{\prime} \mathrm{W} ; 19$ & 0.4 \\
\hline P-6-1 & $0.70880 \pm 1$ & & Tufa & $17^{\circ} 59^{\prime} \mathrm{S} ; 67^{\circ} 03^{\prime} \mathrm{W}$ & \\
\hline C-8-1 & $0.70876 \pm 2$ & & Tufa & $19^{\circ} 19^{\prime} \mathrm{S} ; 68^{\circ} 22^{\prime} \mathrm{W}$ & \\
\hline$C-8-2$ & $0.70878 \pm 2$ & & Tufa & $19^{\circ} 19^{\prime} \mathrm{S} ; 68^{\circ} 22^{\prime} \mathrm{W}$ & \\
\hline E-2-1 & $0.70877 \pm 1$ & & Tufa & $20^{\circ} 14^{\prime} \mathrm{S} ; 68^{\circ} 28^{\prime} \mathrm{W}$ & \\
\hline$C-2-2$ & & & Tufa & $19^{\circ} 40^{\prime} \mathrm{S} ; 68^{\circ} 06^{\prime} \mathrm{W} ; 13$ & -2.0 \\
\hline Chita-2 & & & Tufa & $20^{\circ} 06^{\prime} \mathrm{S} ; 66^{\circ} 58^{\prime} \mathrm{W} ; 20 \mathrm{~B}$ & -0.8 \\
\hline U-7-1 & & & Tufa & $20^{\circ} 43^{\prime} \mathrm{S} ; 67^{\circ} 59^{\prime} \mathrm{W} ; 29$ & -0.4 \\
\hline U-4-1 & & & Tufa & $20^{\circ} 05^{\prime} \mathrm{S} ; 68^{\circ} 13^{\prime} \mathrm{W} ; 17$ & 3.4 \\
\hline S-6-1 & & & Tufa & $19^{\circ} 50^{\prime} \mathrm{S} ; 67^{\circ} 09^{\prime} \mathrm{W} ; 14$ & -1.5 \\
\hline S-3-1 & & & Tufa & $19^{\circ} 50^{\prime} \mathrm{S} ; 67^{\circ} 09^{\prime} \mathrm{W} ; 14$ & 0.6 \\
\hline S-20-1 & & & Tufa & $19^{\circ} 50^{\prime} \mathrm{S} ; 67^{\circ} 09^{\prime} \mathrm{W} ; 14$ & -2.8 \\
\hline Average & $0.70881 \pm 3$ & $1150 \pm 215$ & & & $-1.1 \pm 1.3$ \\
\hline
\end{tabular}


Table 2 (continued)

\begin{tabular}{|c|c|c|c|c|c|}
\hline Sample/lake cycle & ${ }^{87} \mathrm{Sr} /{ }^{86} \mathrm{Sr}( \pm 1 \mathrm{SE})$ & Sr ppm $( \pm 1 \mathrm{SE})$ & Sample material & Sample location (latitude; longitude; site \# ${ }^{\mathrm{a}}$ ) & $\delta^{18} \mathrm{O}^{\mathrm{b}}(\%)$ \\
\hline \multicolumn{6}{|l|}{ Tauca regression } \\
\hline B-12 & $0.70878 \pm 1$ & $1067 \pm 0.05$ & Tufa & $19^{\circ} 51^{\prime} \mathrm{S} ; 67^{\circ} 33^{\prime} \mathrm{W} ; 15$ & $0.1 ;-0.1$ \\
\hline B-14 & $0.70880 \pm 1$ & $1099 \pm 0.05$ & Tufa & $19^{\circ} 51^{\prime} \mathrm{S} ; 67^{\circ} 33^{\prime} \mathrm{W} ; 15$ & $-0.1 ;-0.71$ \\
\hline S-20-1 & $0.70878 \pm 1$ & & Cemented gravel & $19^{\circ} 50^{\prime} \mathrm{S} ; 67^{\circ} 09^{\prime} \mathrm{W} ; 14$ & -2.8 \\
\hline Average & $0.70879 \pm 1$ & & & & $-0.7 \pm 1.2$ \\
\hline \multicolumn{6}{|l|}{ Sajsi } \\
\hline S-17-7 & $0.70883 \pm 1$ & & Tufa & $19^{\circ} 50^{\prime} \mathrm{S} ; 67^{\circ} 09^{\prime} \mathrm{W} ; 14$ & -1.1 \\
\hline S-17-4 & & & Tufa & $19^{\circ} 50^{\prime} \mathrm{S} ; 67^{\circ} 09^{\prime} \mathrm{W} ; 14$ & -3.1 \\
\hline S-17-3 & $0.70888 \pm 1$ & & Tufa & $19^{\circ} 50^{\prime} \mathrm{S} ; 67^{\circ} 09^{\prime} \mathrm{W} ; 14$ & \\
\hline S-17-1 & $0.70884 \pm 1$ & $688 \pm 1$ & Ooids & $19^{\circ} 50^{\prime} \mathrm{S} ; 67^{\circ} 09^{\prime} \mathrm{W} ; 14$ & -3.8 \\
\hline S-17-2 & $0.70876 \pm 1$ & & Ooids & $19^{\circ} 50^{\prime} \mathrm{S} ; 67^{\circ} 09^{\prime} \mathrm{W} ; 14$ & -3.5 \\
\hline Average & $0.70883 \pm 5$ & & & & $-2.9 \pm 1.2$ \\
\hline \multicolumn{6}{|l|}{ Inca Huasi } \\
\hline U-28-1 & $0.70882 \pm 1$ & & Tufa & $19^{\circ} 57^{\prime} \mathrm{S} ; 68^{\circ} 15^{\prime} \mathrm{W} ; 16$ & -0.5 \\
\hline U-27a-1 & $0.70887 \pm 1$ & $2191 \pm 3$ & Tufa & $20^{\circ} 09^{\prime} \mathrm{S} ; 67^{\circ} 49^{\prime} \mathrm{W} ; 19$ & 1.2 \\
\hline Average & $0.70884 \pm 3$ & & & & $0.4 \pm 1.2$ \\
\hline \multicolumn{6}{|l|}{ Salinas } \\
\hline $\mathrm{U}-23 \mathrm{~b}-1 \mathrm{~b}$ & $0.70882 \pm 1$ & & Tufa & $19^{\circ} 41^{\prime} \mathrm{S} ; 67^{\circ} 39^{\prime} \mathrm{W}$ & \\
\hline U-23b-2 & $0.70882 \pm 1$ & & Tufa & $19^{\circ} 41^{\prime} \mathrm{S} ; 67^{\circ} 39^{\prime} \mathrm{W}$ & \\
\hline U-22-1 & $0.70872 \pm 1$ & & Tufa & $19^{\circ} 41^{\prime} \mathrm{S} ; 67^{\circ} 39^{\prime} \mathrm{W} ; 11$ & -1.3 \\
\hline U-22-2 & $0.70884 \pm 1$ & & Tufa & $19^{\circ} 41^{\prime} \mathrm{S} ; 67^{\circ} 39^{\prime} \mathrm{W} ; 11$ & -0.5 \\
\hline U-22-3 & $0.70887 \pm 1$ & & Tufa & $19^{\circ} 41^{\prime} \mathrm{S} ; 67^{\circ} 39^{\prime} \mathrm{W} ; 11$ & -2.2 \\
\hline U-22-4 & $0.70888 \pm 1$ & & Tufa & $19^{\circ} 41^{\prime} \mathrm{S} ; 67^{\circ} 39^{\prime} \mathrm{W} ; 11$ & -0.5 \\
\hline U-22-5 & & & Tufa & $19^{\circ} 41^{\prime} \mathrm{S} ; 67^{\circ} 39^{\prime} \mathrm{W} ; 11$ & 0.4 \\
\hline U-26-1 & $0.70872 \pm 1$ & & Tufa & $20^{\circ} 48^{\prime} \mathrm{S} ; 67^{\circ} 39^{\prime} \mathrm{W} ; 24$ & -0.6 \\
\hline U-26-2 & $0.70885 \pm 1$ & & Tufa & $20^{\circ} 48^{\prime} \mathrm{S} ; 67^{\circ} 39^{\prime} \mathrm{W} ; 24$ & -0.4 \\
\hline U-26-3 & $0.70883 \pm 1$ & $2041 \pm 3$ & Tufa & $20^{\circ} 48^{\prime} \mathrm{S} ; 67^{\circ} 39^{\prime} \mathrm{W} ; 24$ & -0.2 \\
\hline U-26-4 & $0.70876 \pm 1$ & & Tufa & $20^{\circ} 48^{\prime} \mathrm{S} ; 67^{\circ} 39^{\prime} \mathrm{W} ; 24$ & \\
\hline U-31-1 & $0.70885 \pm 1$ & $2637 \pm 4$ & Tufa & $20^{\circ} 14^{\prime} \mathrm{S} ; 67^{\circ} 38^{\prime} \mathrm{W} ; 23$ & -0.9 \\
\hline Average & $0.70882 \pm 6$ & $2340 \pm 420$ & & & $-0.7 \pm 0.7$ \\
\hline \multicolumn{6}{|l|}{ Ouki } \\
\hline $\mathrm{T}-3$ & $0.70926 \pm 1$ & $1958 \pm 0.5$ & Tufa & $18^{\circ} 17^{\prime} \mathrm{S} ; 67^{\circ} 32^{\prime} \mathrm{W} ; 2$ & -1.2 \\
\hline T-9 & $0.70943 \pm 1$ & $1851 \pm 0.5$ & Tufa & $18^{\circ} 17^{\prime} \mathrm{S} ; 67^{\circ} 32^{\prime} \mathrm{W} ; 2$ & -1.7 \\
\hline P-11 & $0.70928 \pm 1$ & $1839 \pm 1$ & Tufa & $18^{\circ} 35^{\prime} \mathrm{S} ; 66^{\circ} 56^{\prime} \mathrm{W} ; 4$ & -2.2 \\
\hline P-17 & $0.70926 \pm 1$ & $1657 \pm 0.5$ & Tufa & $18^{\circ} 35^{\prime} \mathrm{S} ; 66^{\circ} 56^{\prime} \mathrm{W} ; 4$ & -3.3 \\
\hline P-5-3 & $0.70929 \pm 1$ & $1874 \pm 1$ & Tufa & $18^{\circ} 43^{\prime} \mathrm{S} ; 66^{\circ} 52^{\prime} \mathrm{W} ; 5$ & $-1.2 ;-0.9$ \\
\hline $\mathrm{T}-13$ & $0.70944 \pm 1$ & $1888 \pm 1$ & Tufa & $18^{\circ} 17^{\prime} \mathrm{S} ; 67^{\circ} 32^{\prime} \mathrm{W} ; 2$ & -2.2 \\
\hline P-7-2 & $0.70928 \pm 1$ & $2184 \pm 3$ & Tufa & $18^{\circ} 00^{\prime} \mathrm{S} ; 67^{\circ} 03^{\prime} \mathrm{W} ; 1$ & \\
\hline C-19-1 & $0.70944 \pm 3$ & & Shells & $19^{\circ} 19^{\prime} \mathrm{S} ; 67^{\circ} 09^{\prime} \mathrm{W} ; 8$ & \\
\hline C-22-1 & $0.70918 \pm 1$ & & Shells & $19^{\circ} 20^{\prime} \mathrm{S} ; 67^{\circ} 11^{\prime} \mathrm{W} ; 8$ & \\
\hline C-21-1 & $0.70932 \pm 1$ & $1368 \pm 4$ & Shells & $19^{\circ} 20^{\prime} \mathrm{S} ; 67^{\circ} 11^{\prime} \mathrm{W} ; 8$ & \\
\hline $\mathrm{T}-24$ & & & Tufa & $18^{\circ} 17^{\prime} \mathrm{S} ; 67^{\circ} 32^{\prime} \mathrm{W} ; 2$ & -2.5 \\
\hline P-8-2 & & & Tufa & $18^{\circ} 00^{\prime} \mathrm{S} ; 67^{\circ} 02^{\prime} \mathrm{W} ; 1 \mathrm{~B}$ & 0.4 \\
\hline B-3 & & & Tufa & $18^{\circ} 17^{\prime} \mathrm{S} ; 67^{\circ} 32^{\prime} \mathrm{W} ; 2$ & -1.6 \\
\hline Average & $0.70932 \pm 9$ & $1830 \pm 240^{\mathrm{d}}$ & & & $-1.6 \pm 1.0$ \\
\hline
\end{tabular}

a Site numbers correspond to site described by Placzek et al. (2006a).

b Analytical error $(1 \sigma)$ on $\delta^{18} \mathrm{O}$ is typically $0.08 \%$ 。

c Excluded from average value.

d Average concentration values only include data from tufas.

Reliable discharge data is unavailable for most of our basin rivers, and hence we create a simple model that assumes that catchment area is proportional to discharge. Our final model improves on this assumption by weighting catchments by the modern west to east decrease in rainfall seen across the Altiplano (Fig. 1B).

Several useful generalizations can be drawn from the output of this simple model. It is apparent that there are two basic regional inputs that

Table 3

Average isotopic values for paleolake carbonates.

\begin{tabular}{llcl}
\hline Lake cycle & $\delta^{234} \mathrm{U} /{ }^{238} \mathrm{U}$ (activity) & $\delta^{18} \mathrm{O}(\%)$ & ${ }^{87} \mathrm{Sr} /{ }^{86} \mathrm{Sr}$ \\
\hline Coipasa & $1.76 \pm 0.02$ & $0.4 \pm 1.00$ & $0.70854 \pm 4$ \\
Tauca & $1.60 \pm 0.03$ & $-0.95 \pm 1.3$ & $0.70881 \pm 3$ \\
Sajsi & & $-2.9 \pm 1.2$ & $0.70883 \pm 5$ \\
Inca Huasi & & $0.4 \pm 1.2$ & $0.70884 \pm 3$ \\
Salinas & $1.67 \pm 0.02$ & $-0.7 \pm 0.7$ & $0.70882 \pm 6$ \\
Ouki & $1.60 \pm 0.04$ & $-1.6 \pm 1.0$ & $0.70932 \pm 9$ \\
\hline
\end{tabular}

ultimately determine the isotopic composition of basin lakes past and present (Fig. 1B). The first region is watersheds draining the relatively radiogenic (higher ${ }^{87} \mathrm{Sr} /{ }^{86} \mathrm{Sr}$ ) rocks of the $\mathrm{EC}\left({ }^{87} \mathrm{Sr} /{ }^{86} \mathrm{Sr}_{\mathrm{ec}}=0.71096\right.$, $f_{\mathrm{Sr}}=0.87$ ). The second region consists of watersheds draining the relatively non-radiogenic (lower ${ }^{87} \mathrm{Sr} /{ }^{86} \mathrm{Sr}$ ) rocks of the WC with an ${ }^{87} \mathrm{Sr}$ / ${ }^{86} \mathrm{Sr}_{\mathrm{wc}}=0.70687$ and $f_{\mathrm{Sr}}=0.13$; this is dominated by the Río Lauca which flows into the Coipasa Basin. Higher ${ }^{87} \mathrm{Sr} /{ }^{86} \mathrm{Sr}$ ratios therefore must mean greater input from the EC and lower ratios greater input from the WC.

$\delta^{18} \mathrm{O}$

We also constructed a $\delta^{18} \mathrm{O}$ evolution model that can be compared to $\delta^{18} \mathrm{O}$ values of modern and ancient lakes. This model is based on that of Craig and Gordon (1965) and predicts changing $\delta^{18} \mathrm{O}$ of lake water with continued evaporation:

$\delta_{L}=\left(\delta_{0}-A / B\right) f^{B}+A / B$ 


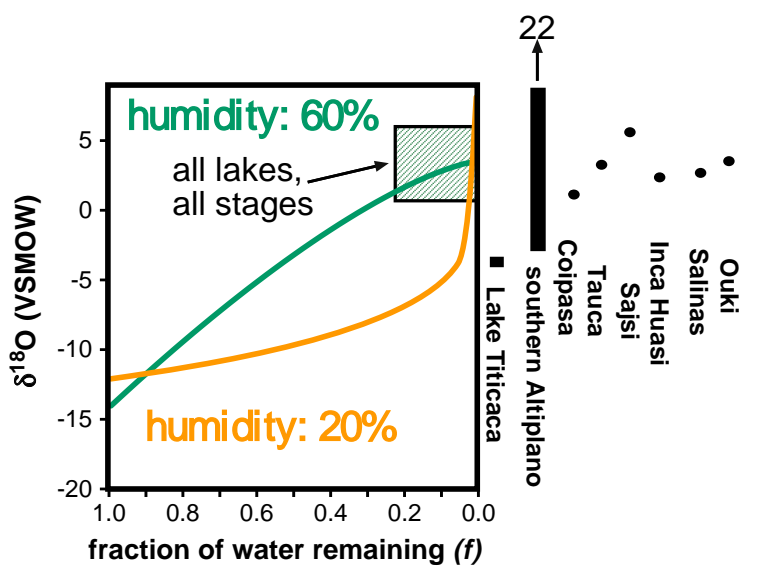

Figure 6. Evaporative model for southern Altiplano lakes. For highly evaporated lakes, changes in $\delta^{18} \mathrm{O}$ due to evaporation are much less for high ( $\left.>50 \%\right)$ relative humidity (black line) than low humidity (gray line; $<50 \%$ ). Initial $\delta^{18} \mathrm{O}$ (VSMOW) values of $-14 \%$ 。 are assumed for the $60 \%$ humidity model, consistent with modern rivers flowing into Titicaca; a value of $-12 \%$ is assumed for the $20 \%$ humidity model (after modeled Taucaphase values from Cross et al., 2001). Both models assume an $\varepsilon_{\text {water-vapor }}=18 \%$ ond temperature $=8^{\circ} \mathrm{C}$. Boxes indicate values of modern waters. Reconstructed paleolake water values (VSMOW) are shown as circles assuming modern temperature $\left(10.8^{\circ} \mathrm{C}\right)$ minus the average temperature change at Vostock (Petit et al., 1999) for each lake cycle.

where,

$A=\frac{h \delta_{A}+\Delta \varepsilon+\varepsilon / \alpha}{1-h+\Delta \varepsilon}$

$B=\frac{h-\Delta \varepsilon-\varepsilon / \alpha}{1-h-\Delta \varepsilon}$

and

$\Delta \varepsilon=14.2(1-h)$

where $\delta_{L}$ is the oxygen isotope composition of the lake water, $f$ is the fraction of water remaining, $\delta_{A}$ is the oxygen isotope composition of the atmosphere, $h$ is the relative humidity, and $\varepsilon$ is the equilibrium enrichment factor, and $\alpha$ is the equilibrium fractionation factor.

As visible in Eqs. (3)-(6), relative humidity is critical in determining the shape of the $\delta^{18} \mathrm{O}$ evolution curve (Fig. 6). Modern lake values that vary over $30 \%$ are associated with both low humidity and extreme evaporative enrichment of modern rainfall inputs of about $-17.5 \%$ o (Cross et al., 2001).

\section{Discussion}

Paleohydrologic implications

The range of ${ }^{87} \mathrm{Sr} /{ }^{86} \mathrm{Sr}$ ratios displayed by the lake carbonates must ultimately be tied to the shifting balance of inputs from the WC (0.70687) and the EC (0.71096). Today, an increased flux from the EC (largely the Poópo Basin) would raise composite lake ratios, whereas an increased flux of WC waters (principally the Coipasa/Uyuni basin) would decrease it. Using the ${ }^{87} \mathrm{Sr} /{ }^{86} \mathrm{Sr}$ ratios of paleolake carbonates, this would mean that the relative WC/EC Sr contribution to modern Lake Titicaca is 0.67 , to the Coipasa lake cycle was 0.60 , to the Ouki lake cycle was 0.41 , and to all other lakes was about 0.53 . Applying this simple model to the modern Titicaca fails to reproduce the modern ${ }^{87} \mathrm{Sr} /{ }^{86} \mathrm{Sr}$ value for Lake Titicaca. The ${ }^{87} \mathrm{Sr} /{ }^{86} \mathrm{Sr}$ ratio of Lake Titicaca produced by this simple model is 0.7106 , much higher than the observed ratio for the lake today of $0.7082-0.7084$.

We can bring observed and modeled ${ }^{87} \mathrm{Sr} /{ }^{86} \mathrm{Sr}$ ratios into better agreement for modern Lake Titicaca by recognizing that catchments in the EC with peaks between 4500 and 6400 m have relative discharges that are much smaller than predicted by their catchment area. Indeed, the average discharge of such catchments (Table 1, \#2, 3, 4, 8, and 9) in the Lake Titicaca basin is $\sim 40 \%$ that of the value predicted by the catchment area (calculated from discharge data in Grove et al., 2003). This likely arises from both orographic effects related to humid air flowing around high peaks of the EC and lake effect precipitation, which is also greater to the west (Roche et al., 1992; Fig. 1B). Our final model uses a weighting factor for catchments in the EC with peaks between 4500 and $6400 \mathrm{~m}$ (Table 1). Adding the assumption that $\mathrm{Sr}$ contributions from Lake Titicaca are consistent with the modern situation ( $\sim 5 \%$ input from Titicaca), our model yields ${ }^{87} \mathrm{Sr} /{ }^{86} \mathrm{Sr}$ ratios and fractional contributions for the Poópo basin of 0.7107 and $f=0.11$, and ${ }^{87} \mathrm{Sr} /{ }^{86} \mathrm{Sr}=0.7082$ and $f=0.84$ for the Uyuni/Coipasa basin (Table 4). This model also produces an ${ }^{87} \mathrm{Sr} /{ }^{86} \mathrm{Sr}$ ratio of 0.70823 for Lake Titicaca, which closely matches observed ratios of 0.708215 to 0.708467 (Table 3; from Grove et al. (2003)). This suggests to us that our estimates of discharge using modified watershed area are valid. Unlike Titicaca waters, ${ }^{87} \mathrm{Sr} /{ }^{86} \mathrm{Sr}$ ratios of the much smaller southern lakes are seasonally variable and probably influenced by rare flood events and salt dissolution (Table 1), making comparisons of modeled and observed ratios more problematic.

Our model reveals that the relatively low ${ }^{87} \mathrm{Sr} /{ }^{86} \mathrm{Sr}$ ratios of waters from the Coipasa/Uyuni basins are balanced by waters with higher ${ }^{87} \mathrm{Sr} /{ }^{86} \mathrm{Sr}$ ratios from the Poópo basin, but for the past we have to consider the possibility that the fractional Titicaca contribution was larger than 5\%, introducing another way, in addition to the WC source, to lever down bulk ${ }^{87} \mathrm{Sr} /{ }^{86} \mathrm{Sr}$ ratios. The treatment of the Titicaca term for the past is the fundamental difference between two previous models of the Sr budget for the southern Altiplano (Coudrain et al.,

Table 4

Lake budgets.

\begin{tabular}{|c|c|c|c|c|c|c|}
\hline Basin & Poópo & Coipasa/Uyuni & Titicaca & Poópo & Coipasa/Uyuni & Titicaca \\
\hline Modern values ( $\mathrm{Sr}$ model) & $0.71073 ; f=11 \%$ & $0.70847 ; f=84 \%$ & $0.70825 ; f=5 \%$ & & & \\
\hline Modern values (water ${ }^{\mathrm{a}}$ ) & & & & $96 \%$ & & $4 \%$ \\
\hline Area (southern Altiplano) & & & & $38 \%$ & $62 \%$ & \\
\hline Area & & & & $28 \%$ & $47 \%$ & $22 \%$ \\
\hline Relative Sr concentration & 0.22 & 1.35 & 0.29 & & & \\
\hline Lake cycle & $\operatorname{Sr}(\%)$ & $\mathrm{Sr}(\%)$ & $\mathrm{Sr}(\%)$ & Water (\%) & Water (\%) & Water $(\%)$ \\
\hline Coipasa & 2.5 & 97.5 & & 10 & 90 & \\
\hline Tauca/Salinas/Inca Huasi/Sajsi & 16 & 80 & $<1$ & 41 & 54 & $4^{\mathrm{a}}$ \\
\hline Ouki A & 39 & 57 & $<1$ & 69 & 27 & $4^{\mathrm{a}}$ \\
\hline Ouki B ${ }^{\mathrm{b}}$ & & 44 & $56^{\mathrm{b}}$ & 31 & & $69^{\mathrm{b}}$ \\
\hline
\end{tabular}

a Assumed values from Coudrain et al. (2002).

b Assuming no contribution from Coipasa/Uyuni, we suggest that actual values are somewhere between the Ouki A and B models, probably closer to the Ouki A model. 
2002; Grove et al., 2003). For the Tauca lake cycle, the Grove et al. (2003) strontium budget model balances modern ${ }^{87} \mathrm{Sr} /{ }^{86} \mathrm{Sr}$ ratios for Lake Titicaca (0.7083) with a composite value of 0.7105 for the entire southern Altiplano determined from $45 \%$ of the watersheds, mostly in the Poópo basin. The Grove et al. (2003) model predicts that $70-83 \%$ of the water for the Tauca lake cycle originated from Lake Titicaca (Grove et al., 2003), a significant contrast to the modern situation where Lake Titicaca accounts for only $5 \%$ of the flux to Lake Poópo (Coudrain et al., 2002).

In our view, the evidence more strongly supports minimal contribution from lake Titicaca, similar to the modern situation. In addition to the evidence we have presented here for an ${ }^{87} \mathrm{Sr} /{ }^{86} \mathrm{Sr}$ ratio for the southern Altiplano that is considerably less than 0.7105 , there are four additional lines of evidence in support of minimal input from Lake Titicaca: (1) values of $\delta^{18} \mathrm{O}$ from paleolake carbonates imply significant rainfall in the southern basins for all lake cycles; (2) hydrologic budget considerations point to minimal input from Lake Titicaca during the Tauca lake cycle; (3) ${ }^{234} \mathrm{U} /{ }^{238} \mathrm{U}$ ratios indicate greater input from the southern basin during the Coipasa lake cycle; and (4) the absence of Coipasa-age shoreline tufas in the Poópo basin, suggests that recharge may have dominantly come from the south. We discuss these lines of evidence as follows.

(1) Average values of $\delta^{18} \mathrm{O}$ (PDB) of carbonates for all paleolake cycles and for all stages within various lake cycles range between -1.6 and $+0.5 \%$. Assuming lake paleotemperatures in the $2-10^{\circ} \mathrm{C}$ range (see caption of Figure 6 for further explanation), $\delta^{18} \mathrm{O}$ (VSMOW) values of paleolake water can be reconstructed and fall between 0 and $5 \%$. We can compare the reconstructed $\delta^{18} \mathrm{O}$ values of paleowaters to values modeled for evaporation under differing humidity conditions. We observe that modeled $\delta^{18} \mathrm{O}$ values of highly evaporated waters converge towards the relatively narrow range of reconstructed paleowater values only under humid conditions (Fig. 6; 60\% humidity). Under less humid conditions, as with today's southern Altiplano where humidity is $<20 \%$, modeled lake water $\delta^{18} \mathrm{O}$ values are higher and more variable than those observed in paleolake carbonates (Fig. 6).

In light of this modeling comparison, two important conclusions can be drawn from the low variance and very positive $\delta^{18} \mathrm{O}$ values of paleolake waters. The very positive values point to highly evaporated lake water, with fraction of water remaining $<0.5$ at all lake stages. The low variance is most consistent with evaporation under much
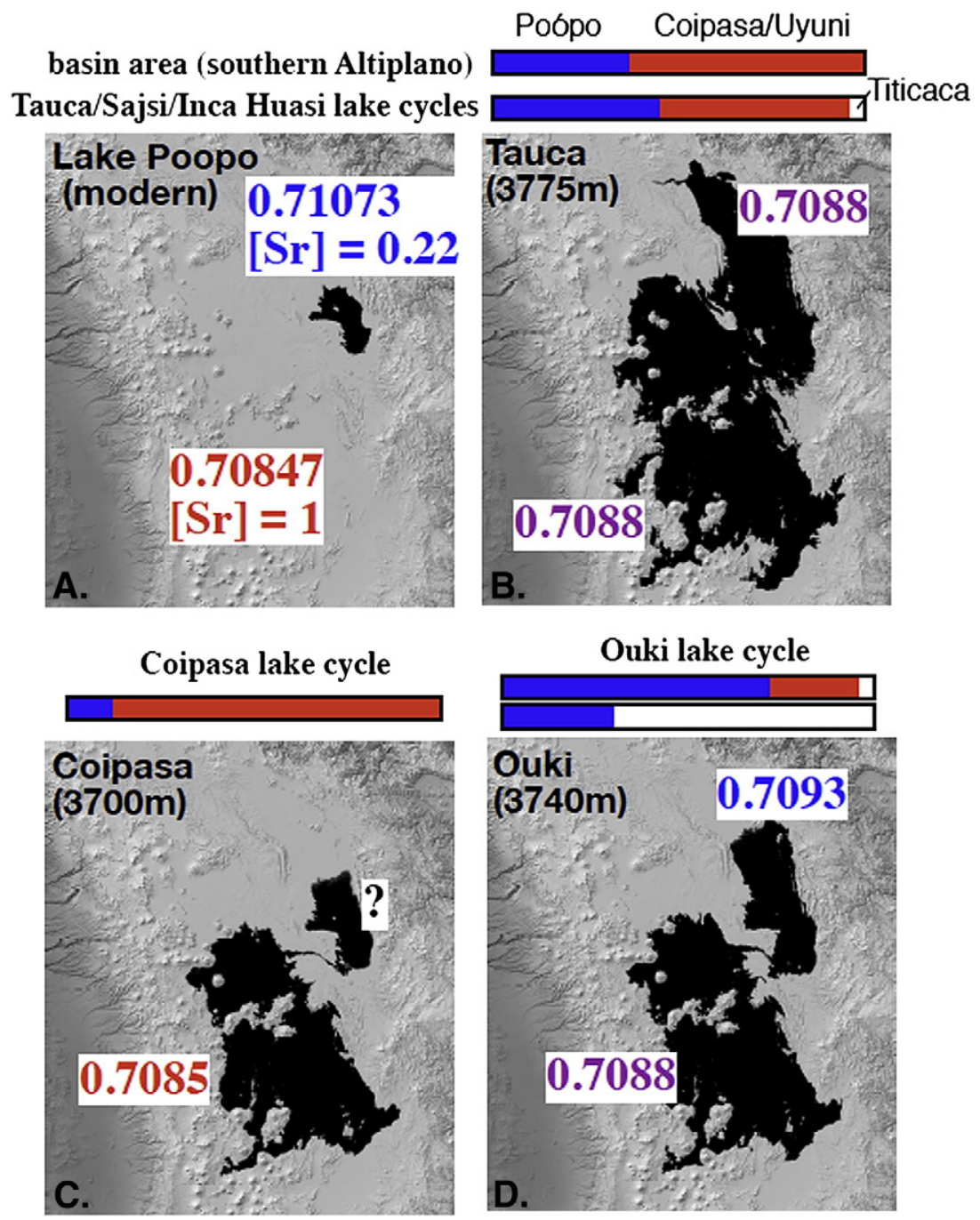

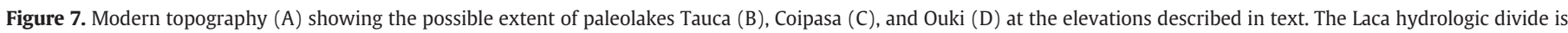

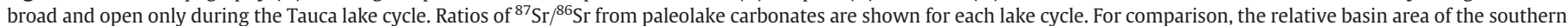

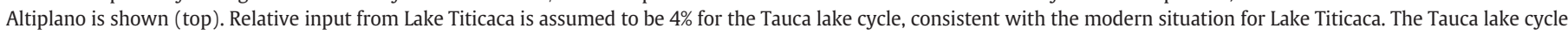

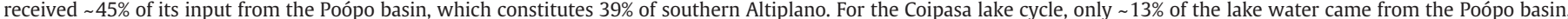

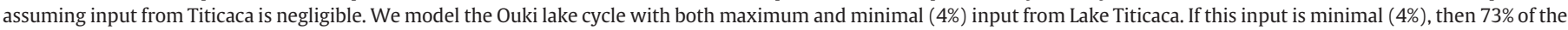

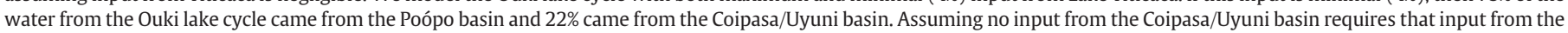
Poópo basin (31\%) be balanced by input from Lake Titicaca (69\%). 
higher humidity ( $60 \%)$ levels than today (20\%), as is graphically visible in Figure 6. The higher humidity in the past implies that more rain than today fell on the southern Altiplano during these lake expansions. These lakes, therefore, were not simply the arid receiving basins of water overflowing from the Titicaca basin.

(2) A variety of hydrologic budget models have been used to reconstruct climate conditions during the Tauca lake cycle (Hastenrath and Kutzbach, 1985; Blodgett et al., 1997; Cross et al., 2001; Condom et al., 2004; Blard et al., 2009). Although these models differ in details, all indicate that evaporation over the considerable surface area of the Tauca lake cycle $\left(\sim 60,000 \mathrm{~km}^{2}\right)$ was huge $\left(>70 \times 10^{9} \mathrm{~m}^{3} / \mathrm{yr}\right)$, comparable to the discharge of major modern rivers such as the Nile or Rhine Rivers. Since maximum paleodischarge along the Río Desaguadero is estimated to be $<20 \times 10^{9} \mathrm{~m}^{3} / \mathrm{yr}$ (Coudrain et al., 2002), it is unrealistic that overflow from Lake Titicaca was sufficient to produce the largest paleolakes.

(3 and 4) Shoreline evidence and ${ }^{234} U /{ }^{238} U$ ratios indicate that the lower ${ }^{87} \mathrm{Sr} /{ }^{86} \mathrm{Sr}$ ratios for the Coipasa lake cycle result from relatively greater contributions from the EC (the Coipasa/Uyuni basin) and not from Lake Titicaca. The Coipasa lake cycle $(12.8-11.4 \mathrm{ka})$ is the youngest lake cycle, and therefore shorelines should be preserved if filling came from Lake Titicaca overflow. Neither Coipasa-age shoreline tufas nor strandlines have been discovered so far in the Poópo basin, suggesting that filling may have been from the south, that is, by northward spilling of the Coipasa/Uyuni Basin. The correspondence between the Coipasa lake cycle highstand and the elevation of the Laca hydrologic divide is also consistent with the Coipasa/Uyuni basin lakes spilling northward into the Poópo basin (Placzek et al., 2009), the reverse of the usual situation today (Fig. 2).

Northward spilling of the Coipasa/Uyuni basins during the Coipasa lake cycle is supported by the very high ${ }^{234} \mathrm{U} /{ }^{238} \mathrm{U}$ ratios of Coipasa- age tufas. Changes in the basin averaged ${ }^{234} \mathrm{U} /{ }^{238} \mathrm{U}$ value of surface waters should reflect changes in the ratio of chemical to physical weathering (Andersen et al., 2009). The ${ }^{234} U /{ }^{238} U$ ratio of surface waters reflects the preferential release of ${ }^{234} U$ during aqueous weathering. The ${ }^{234} \mathrm{U} /{ }^{238} \mathrm{U}$ ratio of surface waters and is strongly tied to soil development; all else being equal, soils formed in dry conditions should be less weathered and retain higher ${ }^{234} \mathrm{U} /{ }^{238} \mathrm{U}$ ratios (Andersen et al., 2009). Thus, the high ${ }^{234} \mathrm{U} /{ }^{238} \mathrm{U}$ ratios and shoreline evidence indicate that the source of low ${ }^{87} \mathrm{Sr} /{ }^{86} \mathrm{Sr}$ ratios for the Coipasa lake cycles is the Coipasa/Uyuni basin, not Lake Titicaca.

The relative contribution of water from the Poópo basin and across the Laca hydrologic divide is the principal factor influencing ${ }^{87} \mathrm{Sr} /{ }^{86} \mathrm{Sr}$ ratios of southern Altiplano paleolakes. The Tauca lake cycle, with an ${ }^{87} \mathrm{Sr} /{ }^{86} \mathrm{Sr}$ value of $\sim 0.7088$, resulted in a deep $(>140 \mathrm{~m}$ ) lake that integrated the Poópo and Coipasa/Uyuni basin. At highstand, a broad $(>70 \mathrm{~m})$, deep $(>50 \mathrm{~m})$ section of the lake covered the Laca hydrologic divide (Figs. 2C, 7). Additionally, ${ }^{87} \mathrm{Sr} /{ }^{86} \mathrm{Sr}$ ratios of $\sim 0.7088$ are observed not only for all stages of the Tauca lake cycle, but also from carbonates of the Sajsi, Inca Huasi, and Salinas lake cycles. Our models suggest that this ratio of $\sim 0.7088$ reflects a marginally greater contribution from the Poópo basin (Fig. 7B), a situation consistent with a very modest north-south precipitation gradient.

Geochemical and field evidence suggests that the Ouki lake cycle filled the Poópo basin to an elevation $>3720 \mathrm{~m}$, and overflowed southward over the Laca hydrologic divide (3700 m) into the Coipasa/ Uyuni basin, where at times much shallower lakes were apparently present (Fig. 2D). Lakes existed in both the Poópo and Coipasa/ Uyuni basin around $\sim 95 \mathrm{ka}$. Sediments in the Poópo basin (Ouki) are at higher elevation $(\sim 3720 \mathrm{~m})$ and have a higher ${ }^{87} \mathrm{Sr} /{ }^{86} \mathrm{Sr}$ ratio $(\sim 0.7093)$ at this time; tufa encrustations in the Uyuni basin (Salinas)

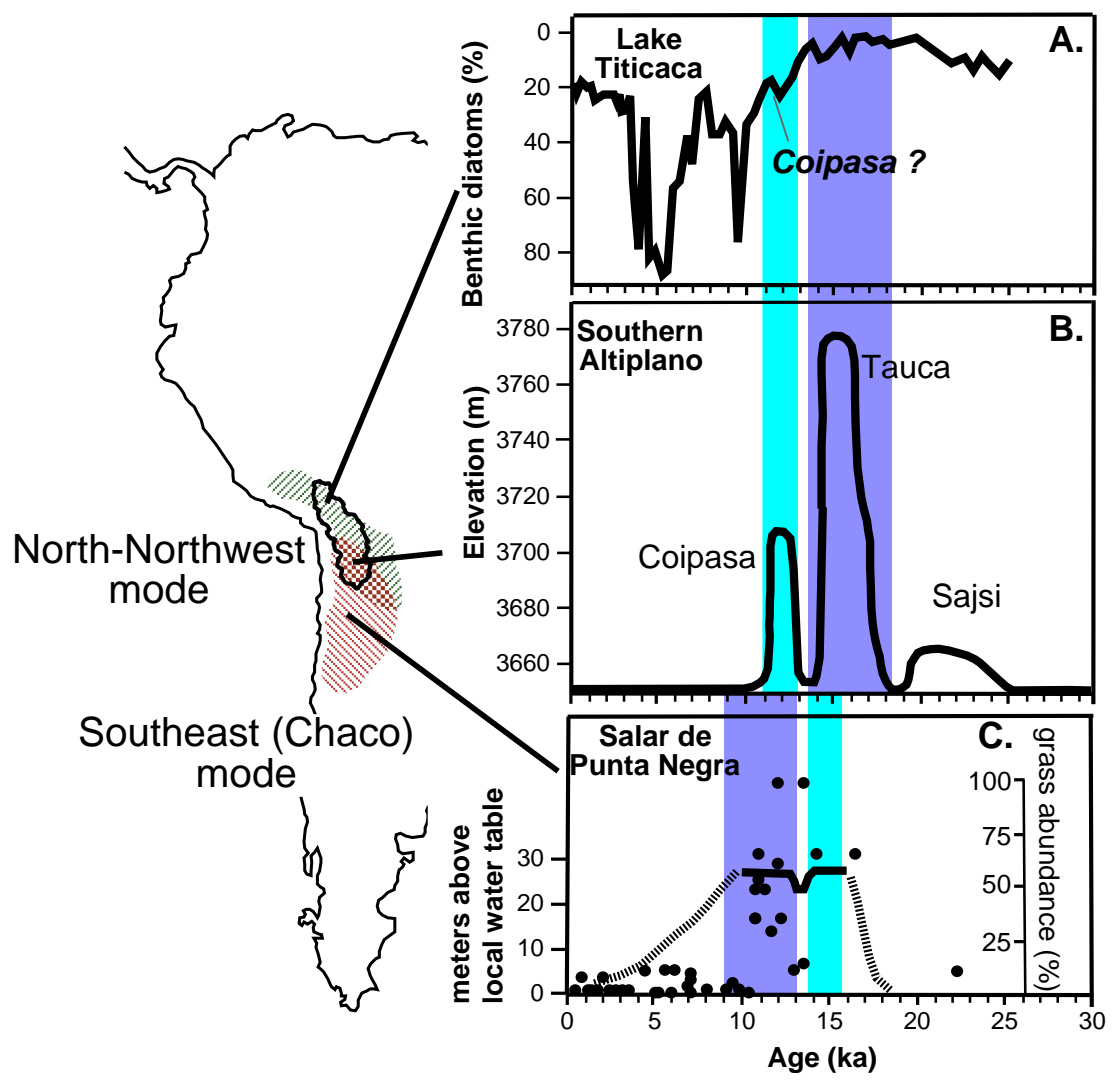

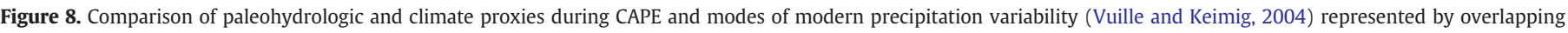

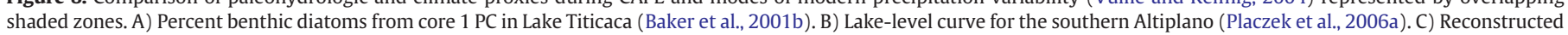
water table height (black line) (Quade et al., 2008) and grass abundance (circles) in rodent middens from the Punta Negra area (Latorre et al., 2002). 
are at much lower elevation $(<3675)$ and have lower $(\sim 0.7088){ }^{87} \mathrm{Sr} /$ ${ }^{86} \mathrm{Sr}$ ratios over the same interval. This implies that although the Poópo basin (Ouki lake cycle) made significant contributions to the Coipasa/Uyuni basin (Salinas lake cycle) during this time, the Coipasa/ Uyuni basin (Salinas lake cycle) made little $(<22 \%)$ or no contribution to the lake (Ouki) in the Poópo basin. Sediments from the Ouki lake cycle consist of thick (generally $>1 \mathrm{~m}$ ) exposures of nearshore carbonates, either sands or massive conical tufa heads. Extensive shoreline sediments indicate prolonged stabilization of the lake by overflow of the Laca hydrologic divide.

\section{Implications for regional climate variability}

Recent work suggests regional variability in the timing and relative magnitude of the two phases of CAPE (Quade et al., 2008). Our new data supports this assertion. The first phase of CAPE (18.1-14.1 ka) produced the largest lake (the Tauca lake cycle) documented on the southern Altiplano and coincides not only with cold conditions in the North Atlantic (Heinrich event 1), but with intense and prolonged La Niña like conditions in the Pacific-both patterns that today bring wet years on the Altiplano. The second phase of CAPE corresponds to the Coipasa lake cycle (12.8-11.4 ka), and on the southern Altiplano is exactly coincidental with the Younger Dryas climate interval, another period of cooling in the North Atlantic. Evidence from rodent middens and wetlands in the Atacama region southeast of Uyuni, however, suggests that to the south the second phase of CAPE (12.8-8 ka) was not only longer-lived, but also wetter than the first phase (18.1$14.1 \mathrm{ka}$ ) (Latorre et al., 2002; Quade et al., 2008). In contrast, the second (Coipasa) phase of CAPE is less pronounced in a lake record from Lake Titicaca (Fig. 8A). One possible explanation for this pattern is that the first phase of CAPE was dominated by the north-northeast mode of rainfall, modulated by both cool North Atlantic temperatures and La Niña-like ocean and atmospheric conditions (Placzek et al., 2006a; Quade et al., 2008), and the second phase of CAPE may be dominated by the southeast (Chaco) mode of rainfall (Quade et al., 2008). Our strontium budget model for the Coipasa lake cycle indicates enhanced precipitation in the southernmost portion of the Altiplano which is consistent with paleo-precipitation that originated from the Chaco region of Argentina instead of from the Amazon. Variability in the Coipasa phase of CAPE must also be tied to cold events in the North Atlantic, and coincidental warming of the southern Atlantic Ocean.

\section{Use of ${ }^{87} \mathrm{Sr} /{ }^{86} \mathrm{Sr}$ ratios as stratigraphic markers}

The unique ${ }^{87} \mathrm{Sr} /{ }^{86} \mathrm{Sr}$ ratios associated with some of the lake cycles make ${ }^{87} \mathrm{Sr} /{ }^{86} \mathrm{Sr}$ ratios very useful stratigraphic tracers. This requires the assumption that lakes are isotopically uniform during any given lake cycle; an assumption that is validated for the Tauca lake cycle by replicated ${ }^{87} \mathrm{Sr} /{ }^{86} \mathrm{Sr}$ of $\sim 0.7088$ at diverse locations around the basins (Table 2). For example, ages from the youngest part of the Tauca lake cycle (18.1-14.1 ka) overlap at $2 \sigma$ with Coipasa-age material (12.8$11.4 \mathrm{ka})$. In the absence of stratigraphic evidence, how do we distinguish them? Deposits from the Coipasa lake cycle consist of fresh, thin $(<0.5 \mathrm{~m})$ tufa crusts at low and moderate elevations $(<3680 \mathrm{~m})$ around the Salars of Coipasa and Uyuni. This carbonate is often superimposed on encrustations from the Tauca lake cycle in a manner that is not obviously disconformable (Fig. 9). The differences in ${ }^{87} \mathrm{Sr} /{ }^{86} \mathrm{Sr}$ ratios between carbonates from the Tauca and Coipasa lake cycles can be used to tie carbonates at the lowest (e.g. U-31-4; Fig. 9) and highest elevations (e.g. Table 2; U-9-6, $3703 \mathrm{~m}$ ) to the Coipasa lake cycle. Thus, both field and geochemical evidence now suggest that the Coipasa lake cycle occurred between 12.8 and 11.4 ka and had a highstand at $\sim 3700 \mathrm{~m}$.

Our models predict that the unique ratios associated with various lake cycles should be recorded in all lacustrine carbonates on a given side of the Laca hydrologic divide. Sub-basins divided by the hydrologic divide will have the same ${ }^{87} \mathrm{Sr} /{ }^{86} \mathrm{Sr}$ ratios only when the Laca hydrologic divide is a broad, deep hydrologic connection, as was the situation during the Tauca lake cycle (Figs. 2, 7). In contrast, different values are observed in the Uyuni (Salinas lake cycle) and Poópo basins (Ouki lake cycle) at $~ 95$ ka. To what extent the Ouki-age (120-95 ka) lake reached the Coipasa/Uyuni basin is the key remaining unknown. Stratigraphic evidence suggests that this extent was possibly limited: no high elevation tufas of Ouki-age have been dated outside the Poópo basin. A test of this would be the analysis of ${ }^{87} \mathrm{Sr} /{ }^{86} \mathrm{Sr}$ ratios of carbonates or salts from cores in the Uyuni basin. ${ }^{87} \mathrm{Sr} /{ }^{86} \mathrm{Sr}$ ratios of about 0.7093 would demonstrate that overflow across the Laca hydrologic divide was substantial enough to dominate southern basin waters during Ouki time. Similarly, during Coipasa

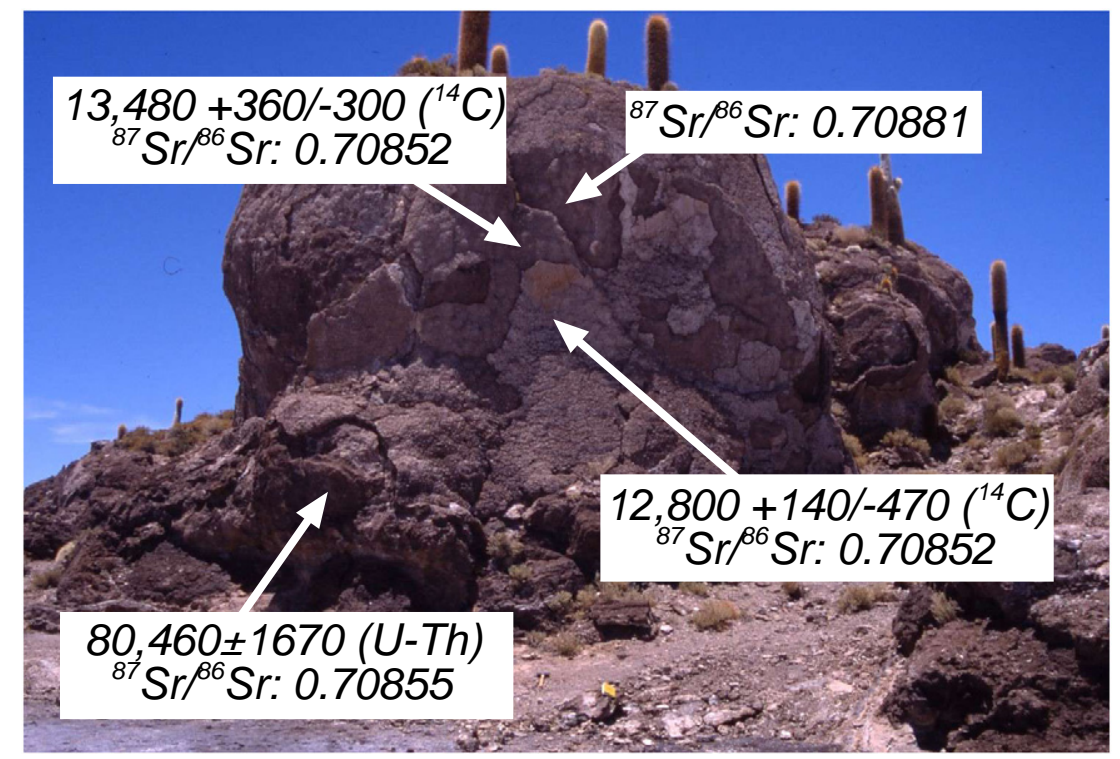

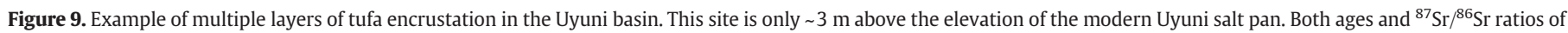
the youngest crusts are consistent with the transgression of the Coipasa lake cycle. Older crusts represent the Tauca and Salinas lake cycles and have ${ }^{87} \mathrm{Sr} /{ }^{86} \mathrm{Sr}=0.7088$. 
time (12.8-11.4 ka), ${ }^{87} \mathrm{Sr} /{ }^{86} \mathrm{Sr}$ ratios in Poópo basin carbonates of $\sim 0.7085$ would point to the presence of a lake dominated by waters from the Coipasa/Uyuni basin. Intermediate values point to coincidental lakes in both sub-basins with more limited hydrologic transfer across the Laca hydrologic divide.

\section{Conclusions}

Strontium, uranium, and oxygen isotopic evidence provide key constraints on the late Quaternary lake history of the southern Bolivian Altiplano. The ${ }^{87} \mathrm{Sr} /{ }^{86} \mathrm{Sr}$ system in the southern Altiplano today is controlled by the interplay of high ${ }^{87} \mathrm{Sr} /{ }^{86} \mathrm{Sr}$ ratios found in the Poópo basin and waters with low ${ }^{87} \mathrm{Sr} /{ }^{86} \mathrm{Sr}$ ratios in the Coipasa/Uyuni basin, plus or minus modest input from Lake Titicaca. We propose that the Ouki lake cycle (120-95 ka) was an expansive lake, deepest in the northern (Poópo) basin of the system, and spilling southward. Shoreline deposits from this lake cycle have elevated ${ }^{87} \mathrm{Sr} /{ }^{86} \mathrm{Sr}$ and point to recharge mainly from the Eastern Cordillera bordering the Poópo Basin. During the Tauca lake cycle (18.1-14.1 ka), all the major basins south of Titicaca were integrated into one deep lake $(3770 \mathrm{~m})$, and inflow to the lake came from local recharge in both the Poópo and Coipasa/Uyuni basins combined with a modest contribution from Lake Titicaca overflow. We attribute this pluvial event to intensification of the tropical summer monsoon, related to cooling in the North Atlantic, perhaps coupled with stronger La Niña conditions in the Pacific. We speculate that the Coipasa lake cycle $(12.8-11.4 \mathrm{ka})$, with a transgression coincidental with the Younger Dryas event was sustained mostly from rainfall in the Coipasa/Uyuni basin, causing this southern lake to rise to the elevation of the Laca hydrologic divide and spill northward into the Poópo basin. This points to intensification of the SE (Chaco) mode of interannual variability in the summer monsoon, probably connected to both tropical and extratropical circulation changes brought about by North Atlantic cooling.

Supplementary materials related to this article can be found online at doi:10.1016/j.yqres.2010.08.004.

\section{Acknowledgments}

We thank Sohrab Tawakholi and Servicio Nacional de Geologia y Mineria (SERGEOMIN) for field logistical support; N. English and J. Mendez for field assistance; M. Ducea and A. Reynolds for assistance in the laboratory; and Julio Betancourt for extensive discussions. This work was supported by NSF-EAR-0207850 to J. Quade and P.J. Patchett. C. Placzek is supported by the G.T. Seaborg foundation.

\section{References}

Aceituno, P., 1988. On the functioning of the Southern Oscillation in the South America sector. Part I: surface climate. Monthly Weather Review 116, 505-524.

Andersen, M.B., Erel, Y., Bourdon, B., 2009. Experimental evidence for 234U-238U fractionation during granite weathering with implications for $234 \mathrm{U} / 238 \mathrm{U}$ in natural waters. Geochimica et Cosmochimica Acta 73, 4124-4141.

Argollo, J., Mourguiart, P., 2000. Late Quaternary climate history of the Bolivian Altiplano. Quaternary International 72, 36-51.

Asahara, Y., Tanaka, T., Kamioka, H., Nishimura, A., 1995. Asian continental nature of ${ }^{87} \mathrm{Sr} /{ }^{86} \mathrm{Sr}$ ratios in north central Pacific sediments. Earth and Planetary Science Letters 133, 105-116.

Baker, P.A., Rigsby, C.A., Seltzer, G.O., Fritz, S.C., Lowenstein, T.K., Bacher, N.P., Veliz, C., 2001a. Tropical climate change at millennial and orbital timescales on the Bolivian Altiplano. Nature 409, 698-701.

Baker, P.A., Seltzer, G.O., Fritz, S.C., Dunbar, R.B., Grove, M.J., Tapia, P.M., Cross, S.L., Rowe, H.D., Broda, J.P., 2001b. The history of South American tropical precipitation for the past 25,000 years. Science 291, 640-643.

Benson, L.V., Peterman, Z., 1995. Carbonate deposition, Pyramid Lake subbasin, Nevada: 3. The use of $87 \mathrm{Sr}$ ratios in carbonate deposits (tufas) to determine the hydrologic state of paleolake systems. Palaeogeography Palaeoclimatology Palaeoecololgy $119,201-213$.

Bills, B.J., deSilva, S.L. Currey, D.R., Emenger, R.S., Lillquist, K.D., Donnellan, A. Worden, B., 1994. Hydro-isostatic deflection and tectonic tilting in the central Andes: initial results of a GPS survey of Lake Minchin shorelines. Geophysical Research Letters 21, 293-296.
Blard, P.-H., Lavé, J., Farley, K.A., Fornari, M., Jiménez, N., Ramirez, V., 2009. Late local glacial maximum in the Central Altiplano triggered by cold and locally-wet conditions during the paleolake Tauca episode (17-15 ka, Heinrich 1). Quaternary Science Reviews 28, 3414-3427.

Blodgett, T.A., Lenters, J.D., Isacks, B.L., 1997. Constraints on the Origin of Paleolake Expansions in the Central Andes: Earth Interactions 1. http://ams.allenpress.com/ amsonline/?request-get-archive\&issn-1087-3562.

Cheng, H., Edwards, R.L., Hoff, J., Gallup, C.D., Richards, D.A., Asmerom, Y., 2000. The half-lives of uranium-234 and thorium-230, Chem. Geol. 169, 17-33.

Condom, T., Coudrain, A., Dezetter, A., Brunstein, D., Delclaux, Jean-Emanuel, S., 2004. Transient modeling of lacustrine regressions: two case studies from the Andean Altiplano. Hydrological Processes 18, 2395-2408.

Coudrain, A., Loubet, M., Condom, T., Talbi, A., Ribstein, P., Pouyaud, B., Quintanilla, J., Dieulin, C., Dupre, B., 2002. Données isotopiques (87Sr/86Sr) et changements hydrologiques depuis 15000 ans sur l'Altiplano. Hydrologic Science 47, 293-306.

Craig, H., Gordon, L.I., 1965. Deuterium and oxygen 18 variations in the ocean and marine atmosphere. In: Tongiogi, E., Lishi, V., Pisa, F. (Eds.), Stable Isotopes in Oceanographic Studies and Paleotemperatures. Spoleto, Italy, pp. 9-130.

Cross, S.L., Baker, P.A., Seltzer, G.O., Fritz, S.C., Dunbar, R.B., 2001. Late Quaternary climate and hydrology of tropical South America inferred from an isotopic and chemical model of Lake Titicaca, Bolivia and Peru. Quaternary Research 56, 1-9.

Enfield, D.B., Mayer, D.A., 1997. Tropical Atlantic sea surface temperature variability and its relation to El Nifio-Southern Oscillation. Journal of Geophysical Research 102, 929-945.

Fornari, M., Rischer, F., Feruad, G., 2001. Dating paleolakes in the central Altiplano of Bolivia. Palaeogeography Palaeoclimatology Palaeoecolology 172, 269-282.

Fritz, S.C., Baker, P.A., Lowenstein, T.K., Seltzer, G.O., Rigsby, C.A., Dwyer, G.S., Tapia, P.M., Arnold, K.K., Ku, T.-L., Luo, S., 2004. Hydrologic variation during the last 170,000 years in the southern hemisphere tropics of South America. Quaternary Research 61, 95-104.

Garreaud, R.D., Aceituno, P., 2001. Interannual rainfall variability over the South American Altiplano. Monthly Weather Review 125, 3157-3171.

Grove, M.J., Baker, P.A., Cross, S.L., Rigsby, C.A., Seltzer, G.O., 2003. Application of strontium isotopes to understanding the hydrology and paleohydrology of the Altiplano, BoliviaPeru. Palaeogeography Palaeoclimatolgy Palaeoecology 194, 281-297.

Hart, W.S. Quade, J., Madsen, D.B., Kaufman, D.S. Oviatt, C.G., 2004. The ${ }^{87} \mathrm{Sr} /{ }^{86} \mathrm{Sr}$ ratios of lacustrine carbonates and lake-level history of the Bonneville paleolake system. Geological Society of America Bulletin 116, 1107-1119.

Hastenrath, S., Kutzbach, J., 1985. Late Pleistocene climate and water budget of the South American Altiplano. Quaternary Research 24, 249-256.

Ku, T.-L., Luo, S., Lowenstein, T.K., Li, J., Spencer, R.J., 1998. U-series chronology of lacustrine deposits in Death Valley, California. Quaternary Research 50, 261-275.

Latorre, C., Betancourt, J.L., Rylander, K.A., Quade, J., 2002. Vegetation invasions into absolute desert: a 45,000-yr rodent midden record from the Calama-Salar de Atacama Basins, northern Chile $\left(22-24^{\circ} \mathrm{S}\right)$. Geological Society of America Bulletin 114, 349-366.

Latorre, C., Betancourt, J.L., Arroyo, M.T.K., 2006. Late Quaternary vegetation and climate history of a perennial river canyon in the Rio Salado basin $\left(22^{\circ} \mathrm{S}\right)$ of northern Chile. Quaternary Research 65, 450-466.

Lenters, J.D., Cook, K.H., 1997. On the origin of the Bolivian High and related circulation features of the South American climate. Journal of Atmospheric Science 54, 656-677.

Minchin, J., 1882. Notes of a Journey through part of the Andean Table-Land of Bolivia in 1882. Proceedings or the Royal Geographic Society 4, 671-676.

Montes de Octa, I., 1997. Geografia y Recursos Naturales de Bolivia. Edobol, La Paz. $614 \mathrm{pp}$.

Petit, J.R., Jouzel, J., Raynaud, D., Barkov, N.I., Barnola, J.-M., Basile, I., Chappellaz, J., Davis, M., Delaygue, G., Delmotte, M., Kotlyakov, V.M., Legrand, M., Lipenkov, V.Y., Lorius, C., Pépin, L., Ritz, C., Saltzman, E., Stievenard, M., 1999. Climate and atmospheric history of the past 420,000 years from the Vostock ice core, Antarctica. Nature 399, 429-436.

Placzek, C., Quade, J., Patchett, P.J., 2006a. Geochronology and stratigraphy of Late Pleistocene lake cycles on the Southern Bolivian Altiplano: implications for causes of tropical climate change. Geological Society of America Bulletin 118, 515-532.

Placzek, C., Patchett, P.J., Quade, J., Wagner, J.D.M., 2006b. Strategies for successful U-Th dating of paleolake carbonates: an example from the Bolivian Altiplano. Geochemistry Geophysics Geosystems 7, Q05024.

Placzek, C., Betancourt, J.A., Quade, J., Patchett, P.J., Latorre, C., Rech, J., Holmgren, C., English, N.B., Matmon, A., 2009. Climate in the dry, central Andes over geologic, millennial, and interannual timescales. Annals of the Missouri Botanical Garden 96, 386-397.

Pretti, V.A., Stewart, B.W., 2002. Solute sources and chemical weathering in the Owens Lake watershed, eastern California. Water Resources Research 38, 1127.

Quade, J., Rech, J.A., Betancourt, J.L., Latorre, C., Quade, B., Rylander, K.A., Fisher, T., 2008. Paleowetlands and regional climate change in the central Atacama Desert, northern Chile. Quaternary Research 69, 343-360.

Roche, M.A., Bourges, J., Cortes, J., Mattos, R., 1992. Climatology and hydrology of the Lake Titicaca Basin. In: Dejoux, C., Iltis, A. (Eds.), Lake Titicaca: a Synthesis of Limnological Knowledge. Kluwer Academic Publishers, Dordrecht, Holland, pp. 63-88.

Rondeau, B., 1990. Géochemie istopique et géochronologie des stromatolite lacustres quaternaries de l'Altiplano bolivien. Ph.D. thesis, Université du Québec à Montréal, 100 pp.

Samos, G., 1949. Some observations on exchange of $\mathrm{CO}_{2}$ between $\mathrm{BaCO}_{3}$ and $\mathrm{CO}_{2}$ gas. Science 110, 663-665.

Seltzer, G.O., Rodbell, D.T., Wright, H.E., 2003. Late-quaternary paleoclimates of the southern Tropical Andes and adjacent regions. Palaeogeography Palaeoclimatolgy Palaeoecology 194, 1-3. 
Servant, M., Fontes, J.C., 1978. Les lacs quaternaries des hautes plateaux des Andes boliviennes: Cahier de l'ORSTROM. Série Géologie, 10, pp. 79-97.

Smith, J.A., Seltzer, G.O., Rodbell, D.T., Farber, D.L., Finkel, R.C., 2005. Early local last glacial maximum in the tropical Andes. Science 308, 678-681.

Sylvestre, F., Servant, M., Servant-Vildary, S., Causse, C., Fournier, M., Ybert, J.P. 1999. Lake-level chronology on the southern Bolivian Altiplano $\left(18-23^{\circ} \mathrm{S}\right)$ during late-Glacial time and the early Holocene. Quaternary Research 51, 281-300.

Vuille, M., Hardy, D.R., Braun, C., Keimig, F., Bradley, R.S., 1998. Atmospheric circulation anomalies associated with 1996/1997 summer precipitation events on Sajama ice cap, Bolivia. Journal of Geophysical Research 103, 11191-11204.

Vuille, M., 1999. Atmospheric circulation over the Bolivian Altiplano during dry and wet periods and extreme phases of the Southern Oscillation. International Journal of Climatology 19, 1579-1600.
Vuille, M., Bradley, R.S., Keimig, F., 2000. Interannual climate variability in the Centra Andes and its relation to tropical Pacific and Atlantic forcing. Journal of Geophysical Research 105, 12,447-12,460.

Vuille, M., Keimig, F., 2004. Interannual variability of summertime convective cloudiness and precipitation in the central Andes derived from ISCCP-B3 data. Journal of Climate 17, 3334-3348.

Zech, R., Kull, C., Kubik, P.W., Veit, H., 2007. LGM and Late-glacial glacier advances in the Cordillera Real and Cochabamba (Bolivia) deduced from ${ }^{10} \mathrm{Be}$ surface exposure dating. Climate of the Past 3, 623-635.

Zolá, R.P., Bengtsson, L., 2007. Three methods for determining the area-depth relationship of Lake Poopó, a large shallow lake in Bolivia. Lakes and reservoirs: research and management 12, 275-284.

Zhou, J., Lau, K.-M., 1998. Does a monsoon climate exist over South America? Journal of Climate $11,1020-1040$. 